Research Article

\title{
Sirt1 Protects Endothelial Cells against LPS-Induced Barrier Dysfunction
}

\author{
Weijin Zhang, ${ }^{1,2}$ Yaoyuan Zhang, ${ }^{1}$ Xiaohua Guo, ${ }^{2}$ Zhenhua Zeng, ${ }^{1,2}$ Jie Wu, ${ }^{1,2}$ Yanan Liu, ${ }^{1}$ \\ Jing He, ${ }^{1}$ Ruiting Wang, ${ }^{1}$ Qiaobing Huang, ${ }^{2}$ and Zhongqing Chen ${ }^{1,2}$ \\ ${ }^{1}$ Department of Critical Care Medicine, Nanfang Hospital, Southern Medical University, Guangzhou 510515, China \\ ${ }^{2}$ Guangdong Key Lab of Shock and Microcirculation Research, Department of Pathophysiology, Southern Medical University, \\ Guangzhou 510515, China
}

Correspondence should be addressed to Qiaobing Huang; bing@smu.edu.cn and Zhongqing Chen; zhongqingchen2008@163.com Received 22 May 2017; Revised 16 August 2017; Accepted 12 September 2017; Published 25 October 2017

Academic Editor: Andreas Daiber

Copyright (c) 2017 Weijin Zhang et al. This is an open access article distributed under the Creative Commons Attribution License, which permits unrestricted use, distribution, and reproduction in any medium, provided the original work is properly cited.

\begin{abstract}
Sepsis is a threatening health problem and characterized by microvascular dysfunction. In this study, we verified that LPS caused the downregulation of Sirt1 and the hyperpermeability of endothelial cells. Inhibition of Sirt1 with ex 527 or Sirt1 siRNA displayed a higher permeability, while activation of Sirt1 with SRT1720 reversed the LPS-induced hyperpermeability, formation of fiber stress, and disruption of VE-cadherin distribution. In pulmonary microvascular vein endothelial cells isolated from wild-type mice, Sirt1 was attenuated upon LPS, while Sirt1 was preserved in a receptor of advanced glycation end product-knockout mice. The RAGE antibody could also diminish the downregulation and ubiquitination of Sirt1 in LPS-exposed human umbilical vein endothelial cells. An LPS-induced decrease in Sirt1 activity was attenuated by the RAGE antibody and TLR4 inhibitor. In vivo study also demonstrated the attenuating role of Sirt1 and RAGE knockout in LPS-induced increases in dextran leakage of mesenteric venules. Furthermore, activation of Sirt1 prevented LPS-induced decreases in the activity and expression of superoxide dismutase 2, as well as the increases in NADPH oxidase 4 and reactive oxygen species, while inhibition of Sirtl aggravated the SOD2 decline. It also demonstrated that Sirt1-deacetylated p53 is required for p53 inactivation, which reversed the downregulation of $\beta$-catenin caused by LPS.
\end{abstract}

\section{Introduction}

Sepsis is a fatal health problem and characterized by its complicated infectious pathophysiology [1], variable clinical manifestation [2], high therapeutic cost, and poor eventual prognosis [3]. Sepsis is posing great threat to our health, but we still have known little in the understanding of the occurrence and treatment of sepsis. Though sepsis is elusive, we are gradually getting to realize the participation of microvascular barrier disruption and vascular dysfunction in the pathogenesis and development of sepsis. Vascular endothelial cells (ECs) lining the intima of blood vessels are critical in the regulation of nutrient trafficking and maintenance of microvascular homeostasis. Disruption of the EC barrier leads to vascular hyperpermeability and leakage of albumin and fluid, resulting in tissue edema and potential occurrence of organ dysfunction [4]. A great number of compelling evidences reveal that endothelial dysfunction acts as one of the mechanisms underlying sepsis and preventing vascular extravasation could ameliorate mortality from sepsis through the reinforce of endothelial cytoskeleton and modulation of endothelial junctions $[5,6]$. Sepsis is known to induce acute lung injury accompanied by increased pulmonary microvascular leakage and wet/dry weight ratio [7], hinting that the EC barrier might be a promising approach for more profound understanding of sepsis. Nevertheless, the understanding of the molecular mechanisms remains to be explored.

It is acknowledged that the silent information regulator 2 (SIR2) complex is composed of 4 groups, including group I 
with sirtuin 1 (Sirt1), 2, and 3; group II with Sirt4; group III with Sirt5; and group IV with Sirt6 and 7 [8]. By far, Sirt1 has been the most widely studied and reported. As a nicotinamide adenine dinucleotide- $\left(\mathrm{NAD}^{+}-\right)$dependent histone deacetylase, Sirt1 functions as a master regulator of ageing, apoptosis, and stress response [9, 10]. Sirtl is known to regulate EC functions by targeting a broad spectrum of its substrates, including p53 [11], p66Shc [12], and eNOs [13]. Furthermore, Sirt1 was also demonstrated to have a pivotal role in the defense of inflammation in lipopolysaccharide- (LPS-) induced acute lung injury (ALI). Inhibition of Sirt1 with Sirt1 siRNA diminished the salutary effect of resveratrol on inflammation resistance [14]. Deficiency of Sirt1 increases microvascular inflammation, morbidity, and mortality in early sepsis, whereas the Sirt1 activator reversed the aforementioned effect, indicating that Sirt1 may play a protective role in the development of sepsis [15]. However, these data did not clarify whether Sirtl is beneficial to the EC barrier. If so, the molecular mechanism in which Sirtl exerts the protective role in LPS-induced EC dysfunction also needs to be unveiled. The purpose of this research was to study whether and how Sirt1 affects LPS-sparked EC barrier disruption. Together with this, we also deem Sirt1 as a node of the event through enhancing superoxide dismutase 2 (SOD2) expression and inhibiting NADPH oxidase 4 (NOX4) expression to resist oxidative stress and inactivating p53 to inhibit the loss of junctional protein $\beta$-catenin.

SOD2 is well known to scavenge reactive oxygen species (ROS) and ameliorate ROS-induced EC hyperpermeability [16]. The interplay between Sirt1 and SOD2 has been reported to increase ROS resistance in ECs [17-19]. It was also confirmed that Sirt1 inhibition was engaged in upregulation of NOX4, eventually leading to endothelial dysfunction due to $\mathrm{O}_{2}{ }^{--}$production [20]. Based on these findings, we hypothesized that Sirtl could protect the EC barrier against LPS by the mechanism involving the upregulation of SOD2 and the downregulation of NOX4 to resist ROS generation. Furthermore, the adherence junction (AJ) protein $\beta$-catenin is of great significance for the EC barrier, and the degradation of $\beta$-catenin tends to disrupt the barrier $[21,22]$. p53 has been demonstrated to be critical in the occurrence of brain edema [23], and inactivation of p53 has the potential to prevent the degradation of $\beta$-catenin [24]. p53 was first found as the target of Sirt1, and quantities of studies have revealed the role of Sirt1 in deacetylating p53 and its inactivation $[25,26]$. Hence, we also speculated that Sirt1-deacetylated p53 could be another promising target for the protection of junction proteins and the EC barrier.

\section{Results}

2.1. LPS Increases Hyperpermeability in HUVECs. We incubated human umbilical vein endothelial cells (HUVECs) with LPS at different concentrations for various durations. Permeability coefficient was measured by transendothelial electric resistance (TER) and flux of FITC-dextran. As shown in Figure 1(a), $500 \mathrm{ng} / \mathrm{mL}$ LPS increased EC permeability in a time-dependent manner. We found that the TER level was gradually decreased from $12 \mathrm{~h}$, and it did not become significant until $24 \mathrm{~h}$, which coincided with the FITC-dextran coefficient (Figure 1(b)). Therefore, we used $24 \mathrm{~h}$ as the proper time for LPS-induced barrier dysfunction. Accordingly, we found that LPS induced EC hyperpermeability for $24 \mathrm{~h}$ in a dose-dependent fashion. An increase in FITCdextran flux was observed from $100 \mathrm{ng} / \mathrm{mL}$, and the change was significant at 200 and $500 \mathrm{ng} / \mathrm{mL}$, consistent with TER measurement (Figures 1(c) and 1(d)). Hence, ECs were treated with $500 \mathrm{ng} / \mathrm{mL}$ LPS for $24 \mathrm{~h}$ in the following barrier function detections.

2.2. Sirt1 Protects ECs from LPS-Evoked Hyperpermeability. Next, we explored whether protein expression of Sirt1 was changed after exposure to LPS. As shown in Figure 2(a), treatment of $500 \mathrm{ng} / \mathrm{mL}$ LPS induced a sharp reduction in Sirt 1 expression and it kept at a low level ranging from $1 \mathrm{~h}$ to $24 \mathrm{~h}$, indicating the critical role of Sirt1 in LPS-evoked EC reaction. Next, the result showed that Sirt1 ubiquitination was increased after LPS treatment, suggesting that ubiquitination was responsible for the LPS-induced Sirt1 decrease (Figure 2(b)). To evaluate the role of Sirt1 in LPS-induced hyperpermeability, we firstly examined the influence of the Sirt1 activator SRT1720 and the Sirt1 inhibitor ex527 on Sirt1 activity, as well as the effect of Sirt1 siRNA on Sirt1 protein expression. Figure 2(c) shows a significant increase in Sirt1 activity in HUVECs pretreated with SRT1720 (5 $\mu \mathrm{M}, 24 \mathrm{~h})$ and a decrease in Sirt1 activity in HUVECs pretreated with ex527 (20 $\mu \mathrm{M}, 1 \mathrm{~h})$. Then, Sirtl siRNA was applied, and the result showed that Sirt1 siRNA $(20 \mu \mathrm{M})$ efficiently suppressed the protein expression of Sirt1 (Figure 2(d)).

The protective role of Sirt1 in LPS-induced EC hyperpermeability was then verified by monitoring the monolayer barrier and detecting the morphological alterations of Factin and VE-cadherin. It was revealed that the decrease in TER level induced by LPS was remarkably reversed by both pretreatment and simultaneous treatment with SRT1720, consistent with the reversed decrease in flux of dextran (Figure 3(a)). To confirm the important role of Sirt1, the Sirt1 inhibitor and siRNA were also applied. Ex527 and Sirt1 siRNA could further increase EC permeability, indicating the deteriorating EC barrier for the lack of Sirt1 activity (Figures 3(b) and 3(c)). Afterwards, distribution of F-actin and VE-cadherin was observed. The quiescent cells showed F-actin in a normal condition, characterized by typical and intact outline of the cytoskeleton. LPS treatment caused a redistribution of F-actin with stress fiber formation, which rendered cells to contract towards the center of the cell. However, the formation of stress fiber was attenuated in the application of SRT1720 (Figure 3(d)). Consistently, in response to LPS, VE-cadherin was dissociated and disrupted, which was also reversed by SRT1720 pretreatment (Figure 3(e)).

In vivo (Figure 4), images from microscopy showed little extravasation of dextran in the saline-treated mice. By contrast, the LPS-injected mice showed a remarkable increase in dextran flux outside the vessels, implying the 


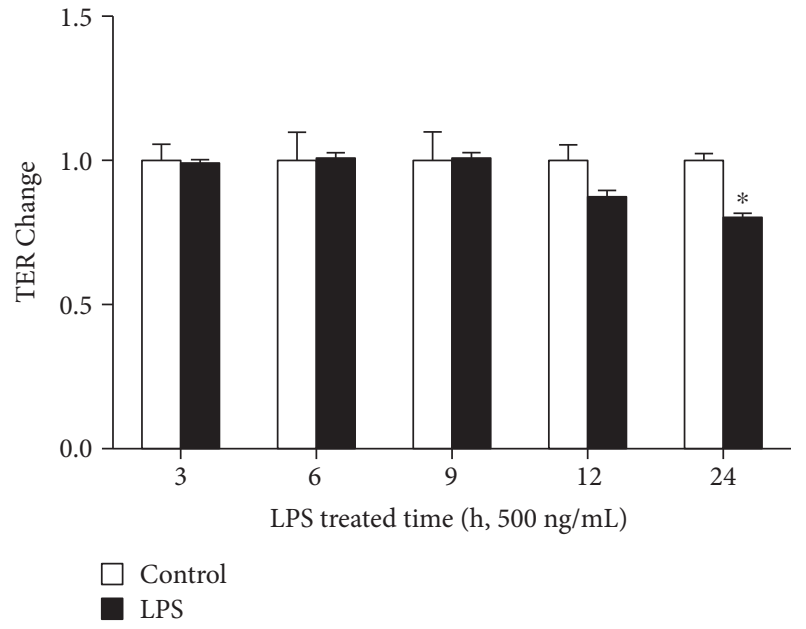

(a)

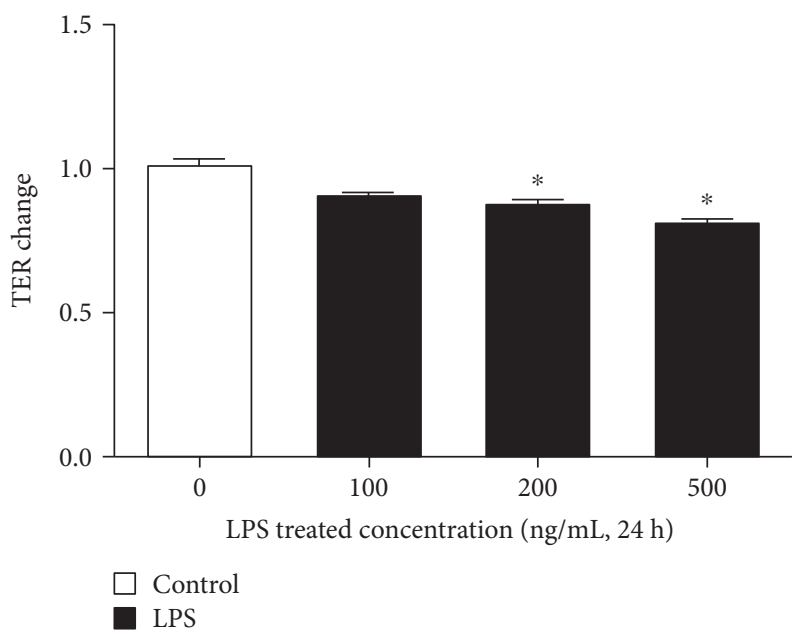

(c)

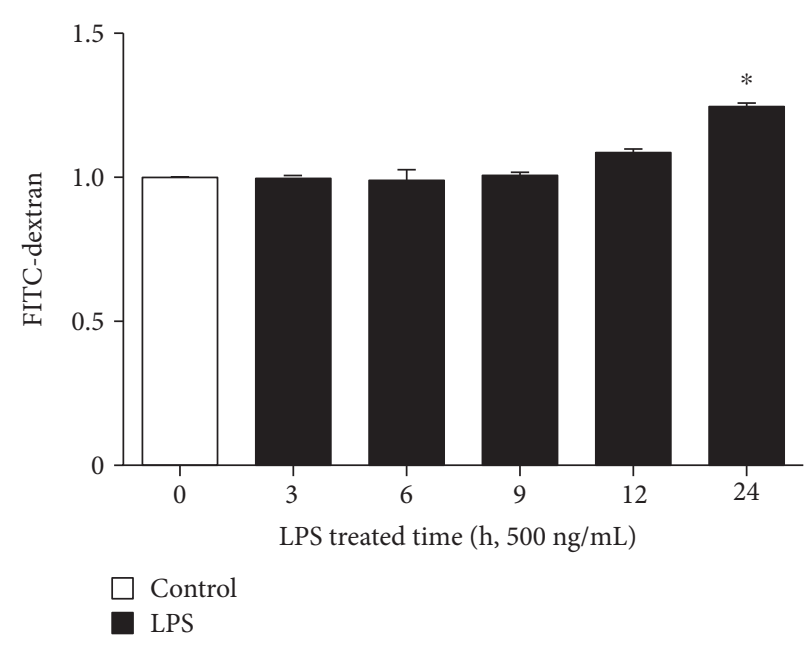

(b)

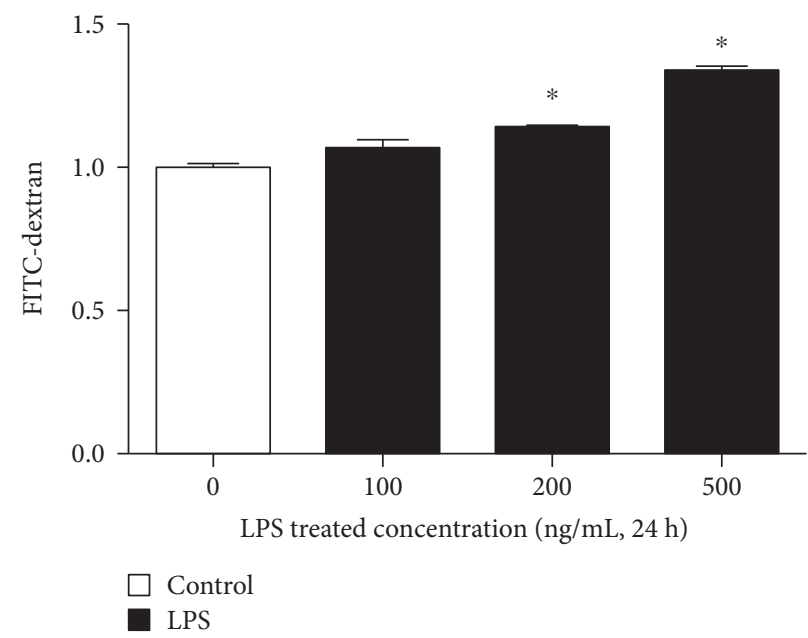

(d)

FIgURE 1: Effects of LPS on EC permeability. Cells were (a, b) treated with $500 \mathrm{ng} / \mathrm{mL}$ of LPS for 3, 6, 9, 12, and $24 \mathrm{~h}$ or with LPS (c, d) at 100 , 200 , and $500 \mathrm{ng} / \mathrm{mL}$ for $24 \mathrm{~h}$, compared to the control with the culture medium. Permeability was assessed by TER and flux of FITC-dextran. $n=3$ per group. ${ }^{*} P<0.05$ versus control.

microvascular hyperpermeability caused by LPS. However, pretreatment of SRT1720 could significantly attenuate the leakage. All these in vivo and in vitro data suggest that Sirt1 played a pivotal role in EC hyperpermeability in response to LPS.

\subsection{RAGE and TLR4 Are Required for LPS-Induced Sirt1} Downregulation. After the critical role of Sirt1 in LPSinduced EC barrier disruption was confirmed, the effect of RAGE on the LPS-evoked Sirt1 decrease was evaluated. In vitro, the RAGE antibody was shown to abolish the increase in Sirt1 ubiquitin and the decrease in Sirtl expression in HUVECs (Figures 2(b) and 5(a)). In pulmonary microvascular vein endothelial cell (PMVECs) of wild-type mice, the expression of Sirt1 was attenuated in response to LPS, while the decrease in Sirtl was reversed in RAGE-knockout mice (Figure 5(b)). Furthermore, the RAGE antibody and the TLR4 inhibitor TAK242 reversed the LPS-induced decrease in Sirt1 activity. These data revealed that RAGE and TLR4 were required for the Sirtl decline concomitantly. The in vivo study also showed that the exudation of dextran from mesenteric venules was decreased in $\mathrm{RAGE}^{-/-}$mice after LPS administration (Figure 4). All these suggested that RAGE and TLR4 were essential for the LPS-sparked Sirt1 decrease and the subsequent hyperpermeability.

2.4. Sirt1 Reverses the Decrease in SOD2 Level and the Increase in NOX4 Level to Resist ROS Induced by LPS. Firstly, we determined the ROS level following LPS ( $500 \mathrm{ng} / \mathrm{mL}, 6 \mathrm{~h}$ ) treatment by using peroxide-sensitive dye $\mathrm{H}_{2}$ DCF-DA. As shown in Figure 6(a), ROS level was significantly enhanced compared to that in the control group, whereas this increase was notably abolished by pretreatment of SRT1720. Together, MDA level was assessed and it was increased in the LPS group, which was reversed in the SRT1720 + LPS group (Figure 6(b)). As $\mathrm{H}_{2} \mathrm{O}_{2}$ could generate more $\mathrm{ROS}$ 


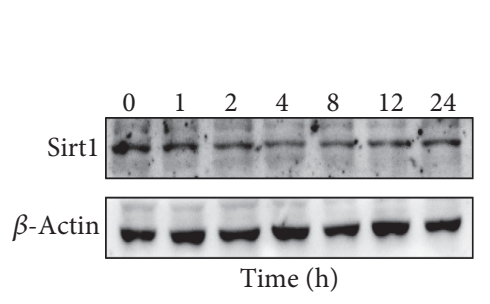

(a)

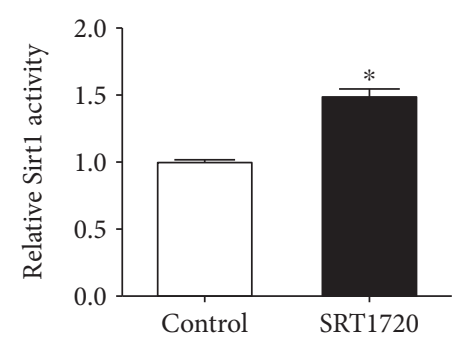

(c)
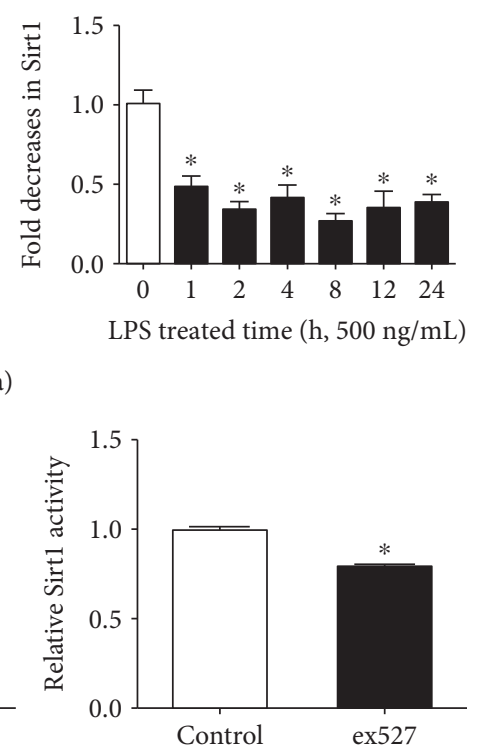

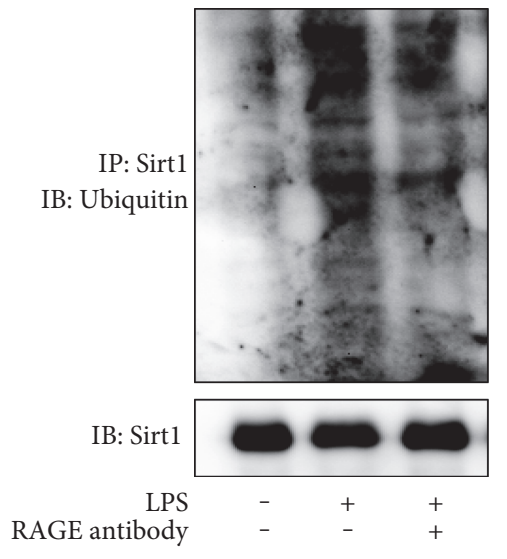

(b)
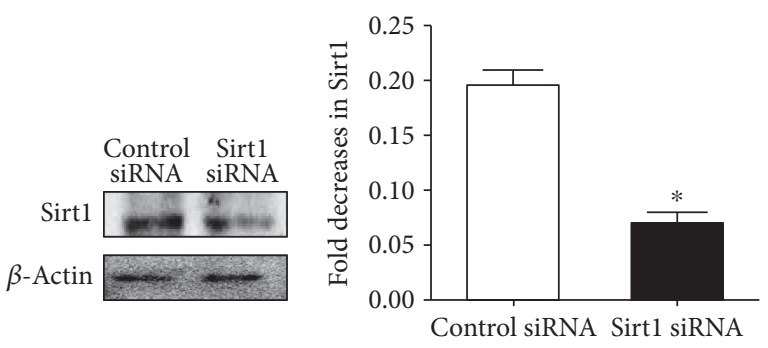

(d)

FIgURe 2: Protein expression of Sirt1 and activity changes in HUVECs. (a) HUVECs were exposed to 500 ng/mL LPS for 1, 2, 4, 8, 12, and $24 \mathrm{~h}$, and protein expression of Sirt1 was detected by WB. $0 \mathrm{~h}$ was used as the control. (b) Cells were stimulated with or without $500 \mathrm{ng} / \mathrm{mL}$ LPS for $1 \mathrm{~h}$ in the presence or absence of the RAGE antibody $(10 \mu \mathrm{g} / \mathrm{mL}, 1 \mathrm{~h})$. The immunoprecipitates were analyzed with anti-Sirt1 and anti-ubiquitin antibodies. (c) Sirt1 activity was examined and SRT1720 enhanced Sirt1 activity, while ex527 inhibited Sirt1 activity. (d) ECs were treated with either control siRNA or Sirtl siRNA targeting Sirt1. The effect of siRNA on the Sirt1 protein expression was measured by WB. $n=3$ per group. ${ }^{*} P<0.05$ versus control or control siRNA.

and antagonize the protective effect of SOD2 on ROS decreases [27], $\mathrm{H}_{2} \mathrm{O}_{2}$ was applied to verify the role of SOD2 in the EC barrier. Figure 6(c) showed that $\mathrm{H}_{2} \mathrm{O}_{2}$ treatment remarkably reversed the protective effect of SRT1720 on LPS-induced barrier disruption.

To elucidate the signal pathways that may respond to Sirt1 activation, HUVECs were stimulated with LPS to detect SOD2 expression. Treatment of $500 \mathrm{ng} / \mathrm{mL}$ LPS significantly caused a sharp decrease in SOD2 expression at $1 \mathrm{~h}$, reaching the bottom at $4 \mathrm{~h}$ and slightly mounting at $8 \mathrm{~h}$ (Figure $7(\mathrm{a})$ ). It was shown that tyrosine nitration of SOD2 was induced by LPS compared with control, leading to SOD2 inactivation (Figure 7(b)). Furthermore, pretreatment of the Sirt1 activator SRT1720 alleviated the decrease in SOD2, while inhibition of Sirt1 with ex527 and siRNA aggravated the SOD2 decline (Figures 7(c), $7(\mathrm{~d})$, and $7(\mathrm{e}))$, which were consistent with the results of SOD activity in different interventions (Figure $7(\mathrm{f})$ ). Then, the NOX inhibitor apocynin was applied to verify the role of NOX in LPS-sparked ROS generation. Figure $7(\mathrm{~g})$ shows that apocynin diminished ROS elevation induced by LPS, indicating NOX as the source of ROS. Pretreatment of SRT1720 alleviated the increase in NOX4 induced by LPS, strengthening the probability that Sirtl may preserve the EC barrier through enhancing
SOD2 expression and attenuating NOX4 level together to resist ROS generation.

2.5. The Activation of Sirt1 and the Inhibition of $p 53$ Prevented the LPS-Evoked Decrease in $\beta$-Catenin Expression. It has been shown that Sirt1 deacetylates and inactivates p53. The p53 inactivation could prevent the degradation of $\beta$-catenin [24]. However, it remains unknown whether there is a relationship between Sirt1 and $\beta$-catenin in a sepsis model. Firstly, to observe the influence of LPS on the change of AJ protein, $500 \mathrm{ng} / \mathrm{mL}$ LPS was exposed to ECs. The time-response experiment (Figure 8(a)) showed a gradual decrease in $\beta$-catenin expression from $0.5 \mathrm{~h}$ to $1 \mathrm{~h}$, indicating that LPS might cause EC hyperpermeability via the reduction in adherent junction proteins. However, downregulation of $\beta$-catenin was abolished due to the application of SRT1720, while pretreatment of ex527 aggravated the downregulation of $\beta$-catenin (Figures $8(\mathrm{~b})$ and $8(\mathrm{c})$ ), which coincided with the effect of SRT1720 and ex527 on LPSinduced barrier disruption (Figures 3(a) and 3(b)). As p53 inhibition has been shown to prevent $\beta$-catenin degradation and p53 was a typical downstream of Sirt1 [25], we next examined the possible role of Sirt1-deacetylated p53 in the increased expression of $\beta$-catenin. Figure $8(\mathrm{~d})$ shows that acetyl-p53 319 was increased in response to 

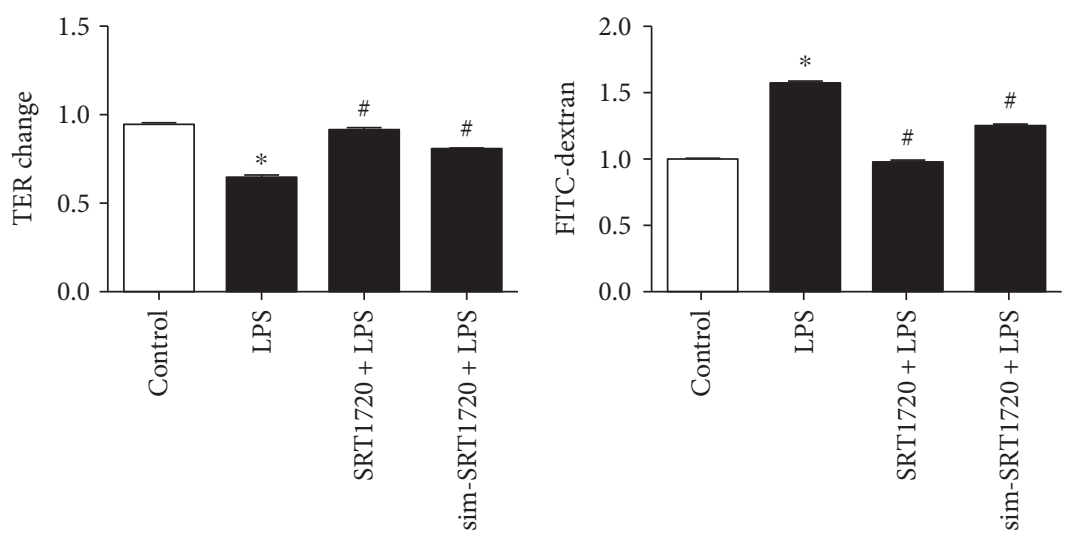

(a)
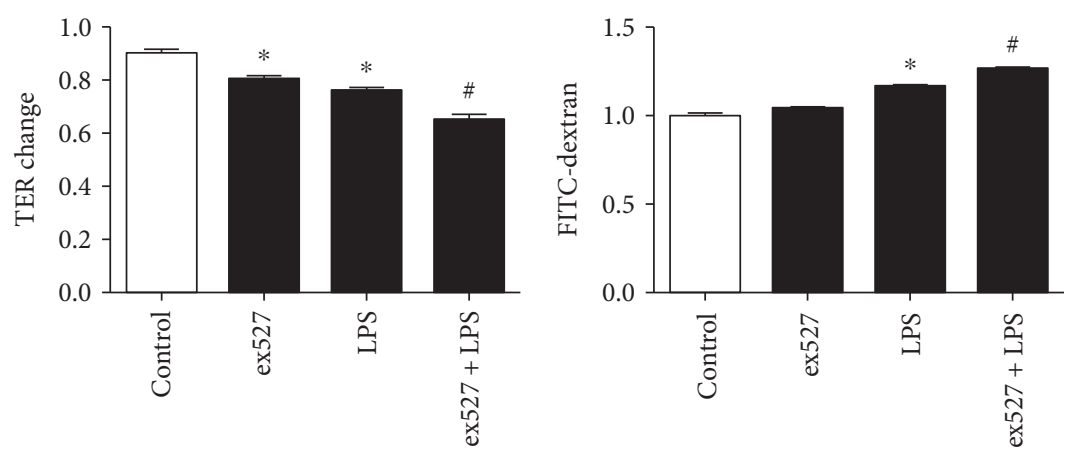

(b)
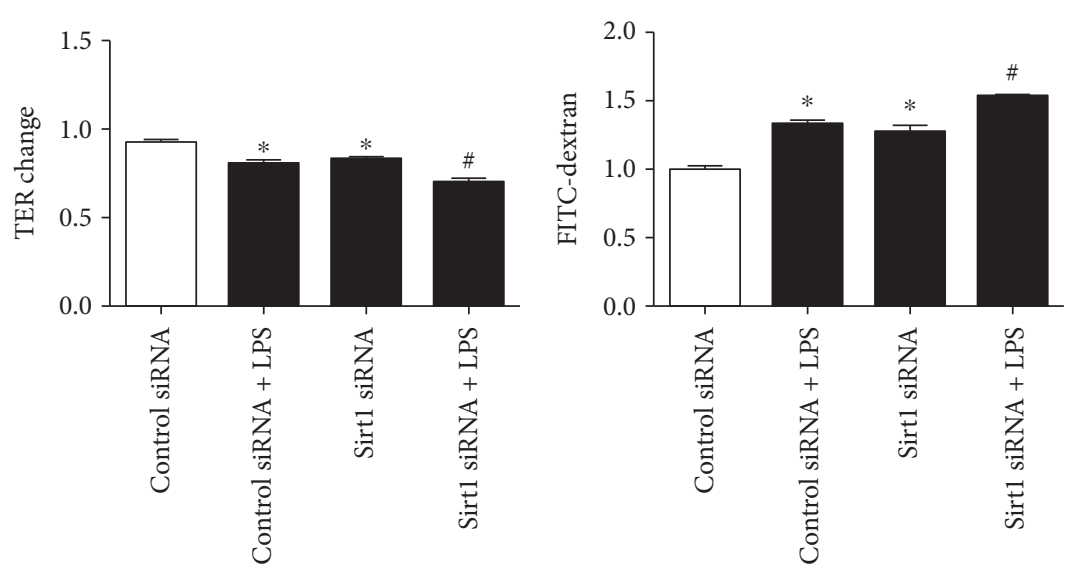
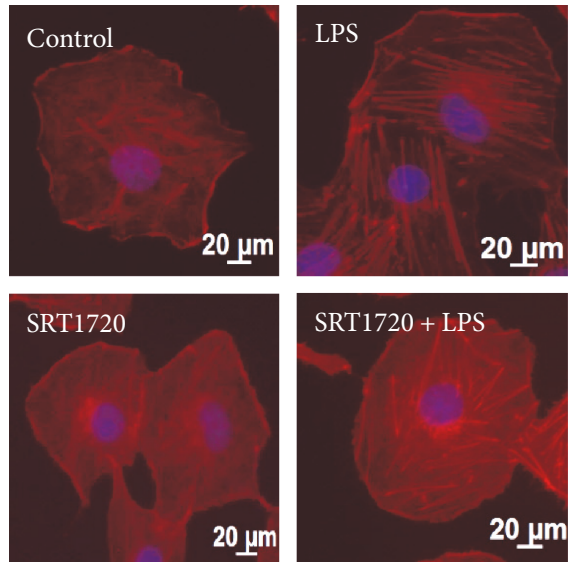

(d)
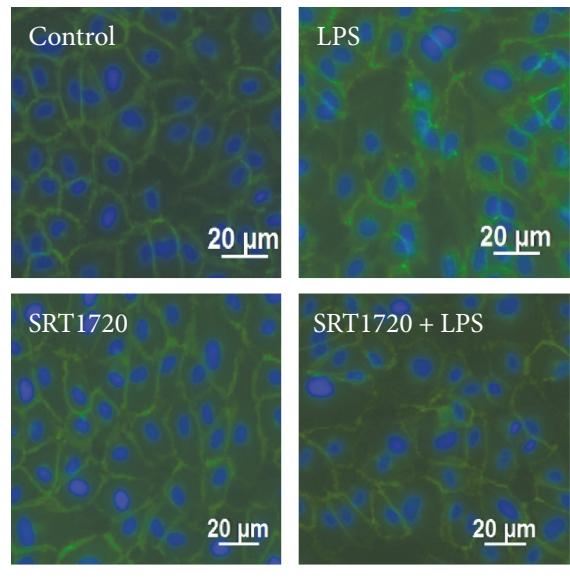

(e)

(c)

FIGURE 3: Protective effect of Sirt1 on LPS-induced EC hyperpermeability. (a) SRT1720 prevented LPS-induced EC hyperpermeability. HUVECs were pretreated with SRT1720 $(5 \mu \mathrm{M}) 24 \mathrm{~h}$ before (SRT1720 + LPS) or at the time (sim-SRT1720 + LPS) of LPS (500 ng/mL, $24 \mathrm{~h}$ ) treatment; then, permeability of monolayers was measured. (b) ex527 exacerbated LPS-evoked EC hyperpermeability. HUVECs were pretreated with ex527 $(20 \mu \mathrm{M}) 1 \mathrm{~h}$ before LPS $(500 \mathrm{ng} / \mathrm{mL}, 24 \mathrm{~h})$ treatment; then, permeability of monolayers was measured. (c) Sirt1 siRNA increased LPS-evoked EC hyperpermeability. Cells treated with control or Sirt1 siRNA were exposed to LPS (500 ng/mL, $24 \mathrm{~h}$ ); then, permeability of monolayers was measured. $n=3$ per group. ${ }^{*} P<0.05$ versus control or control siRNA, ${ }^{\#} P<0.05$ versus LPS or control siRNA + LPS. (d-e) The effect of SRT1720 on the distribution of F-actin and VE-cadherin. Cells were pretreated with SRT1720, followed by examining F-actin and VE-cadherin using confocal microscopy.

$500 \mathrm{ng} / \mathrm{mL}$ LPS for $1 \mathrm{~h}$. However, the acetylated level was attenuated after SRT1720 addition. Significance was not observed concerning total p53 (Supplementary Figure 1 in Supplementary Material available online at https://doi.org/ $10.1155 / 2017 / 4082102)$. The pretreatment of HUVECs with the p53 inhibitor PFT- $\alpha(30 \mu \mathrm{M})$ [28] for $24 \mathrm{~h}$ could also reverse barrier dysfunction induced by LPS in both TER level and the flux of FITC-dextran, implying the important role of p53 in EC hyperpermeability after LPS treatment (Figure $8(\mathrm{e})$ ). Next, PFT- $\alpha$ was used to further specify the role of $\mathrm{p} 53$ in $\beta$-catenin expression. The data showed that the blockade of p53 abolished the reduction in $\beta$-catenin 

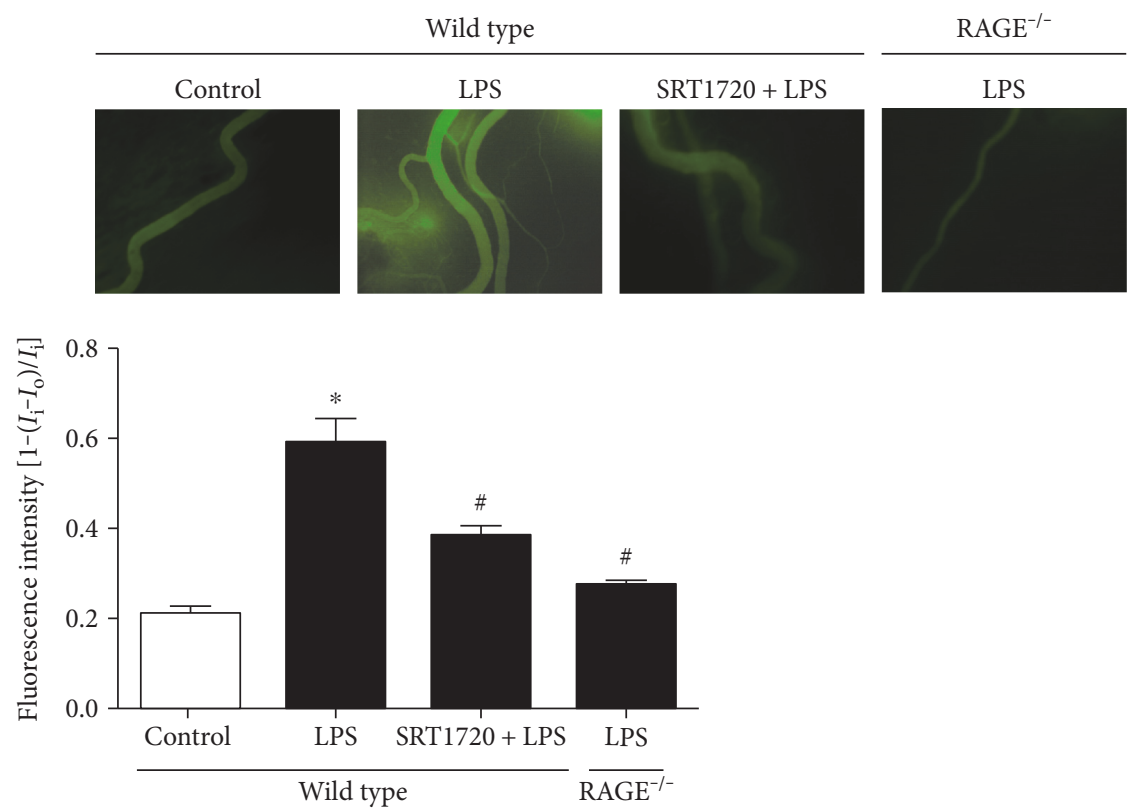

FIGURE 4: Role of Sirt1 and RAGE in microvascular hyperpermeability induced by LPS. Mice were pretreated with SRT1720 or saline via tail vein injection $2 \mathrm{~h}$ prior to LPS treatment. The mice in the LPS group were injected with $15 \mathrm{mg} / \mathrm{kg}$ LPS intraperitoneally, followed by carotid vein cannulation and exudation measurement $6 \mathrm{~h}$ later. Microvascular permeability was determined by the relative dextran density outside the vessels to that inside the vessels. $n=3 .{ }^{*} P<0.05$ versus control, ${ }^{\sharp} P<0.05$ versus wild type with LPS treatment.
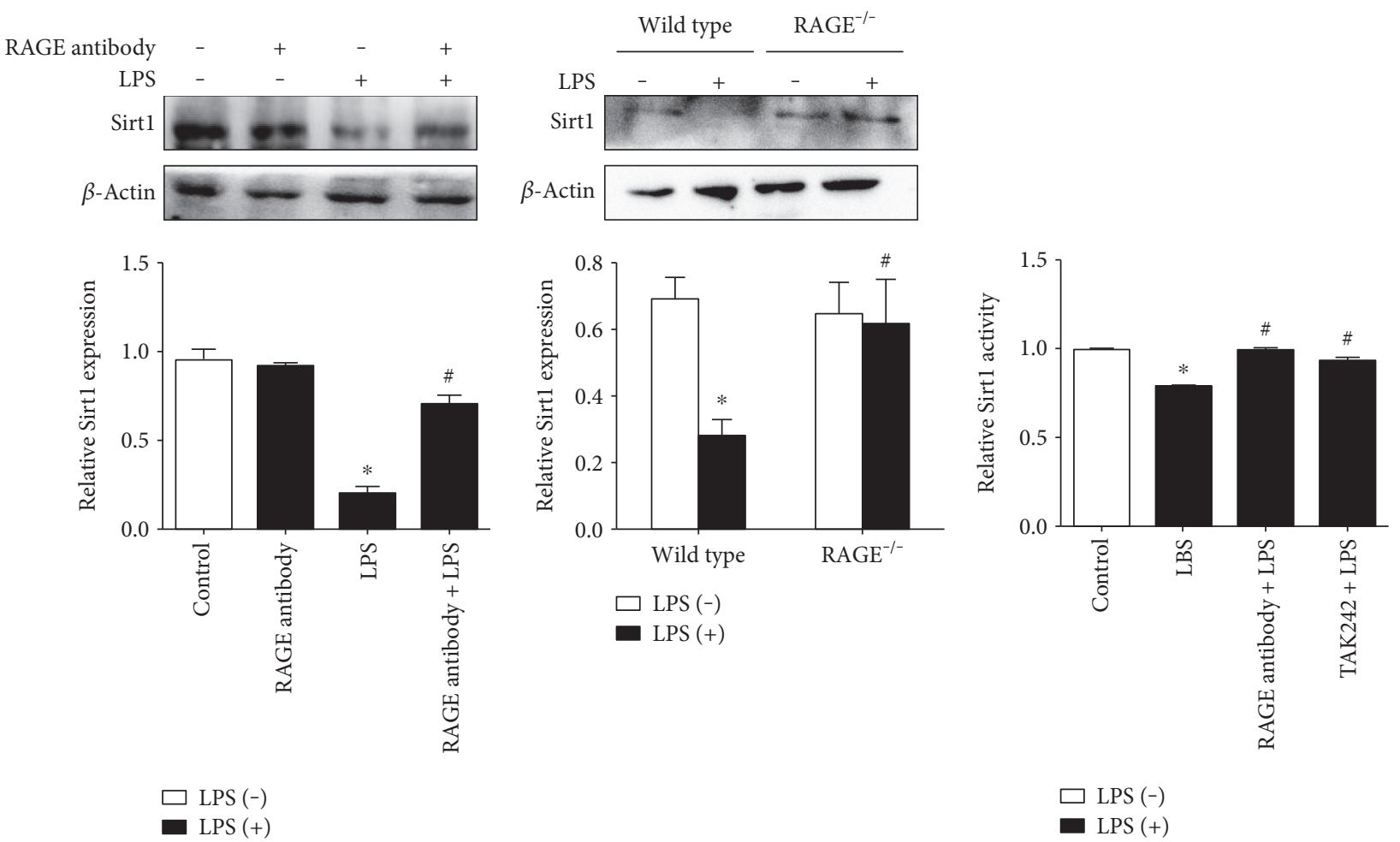

(a)

(b)

(c)

FIGURE 5: Involvement of RAGE and TLR4 in the LPS-induced Sirt1 decrease in ECs. (a) Pretreatment of the RAGE antibody (10 $\mu$ g/mL) for $1 \mathrm{~h}$ attenuated Sirtl decreases in HUVECs. (b) Knockout of RAGE attenuated the LPS-induced Sirt1 decrease in PMVECs. PMVECs of knockout mice and wild-type mice were incubated with or without LPS. Expression of Sirt1 was evaluated by WB. (c) Pretreatment of the RAGE antibody $(10 \mu \mathrm{g} / \mathrm{mL})$ and TLR4 inhibitor $(3 \mu \mathrm{M})$ for $1 \mathrm{~h}$ attenuated the LPS-induced decrease in Sirt1 activity in HUVECs. $n=3$. ${ }^{*} P<0.05$ versus control or wild type without LPS treatment, ${ }^{*} P<0.05$ versus LPS or wild type with LPS treatment. 

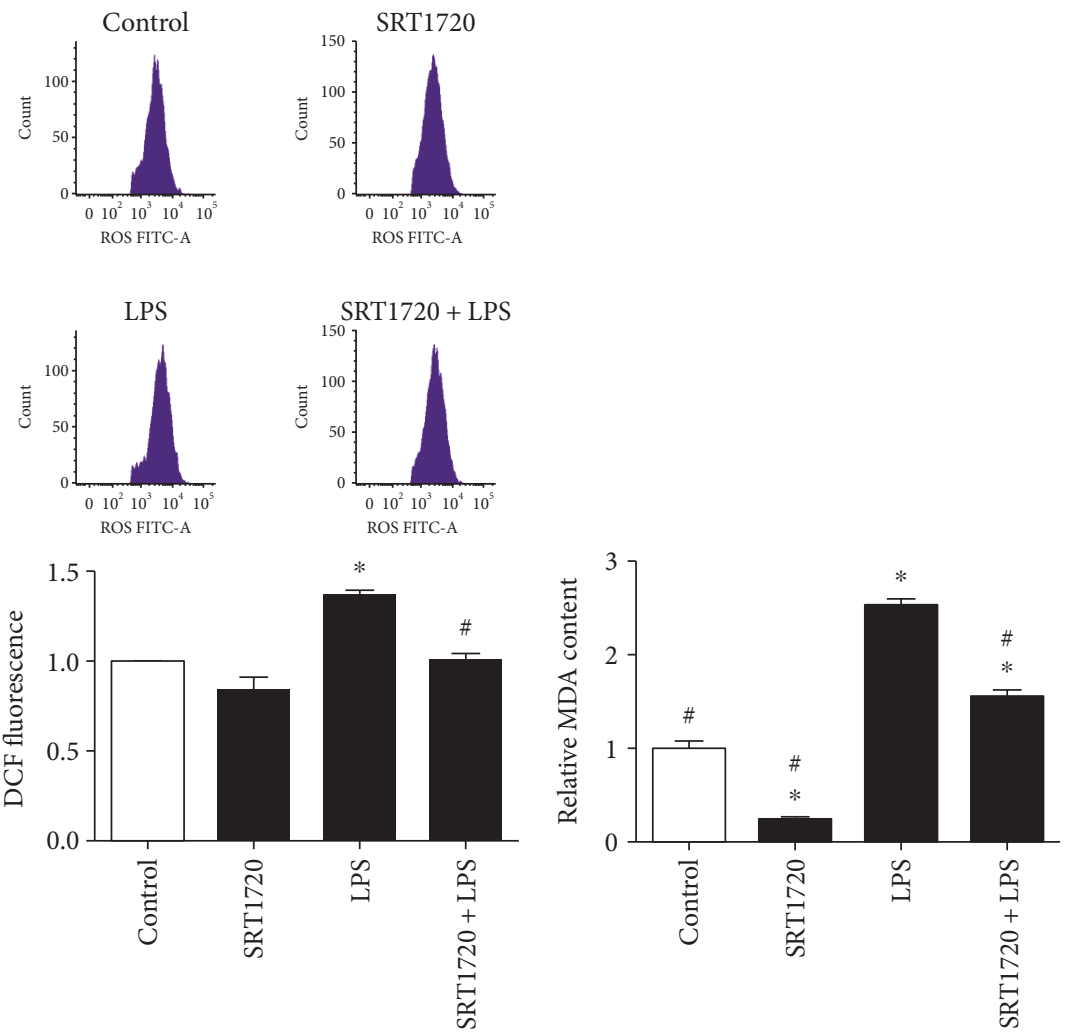

(a)

(b)
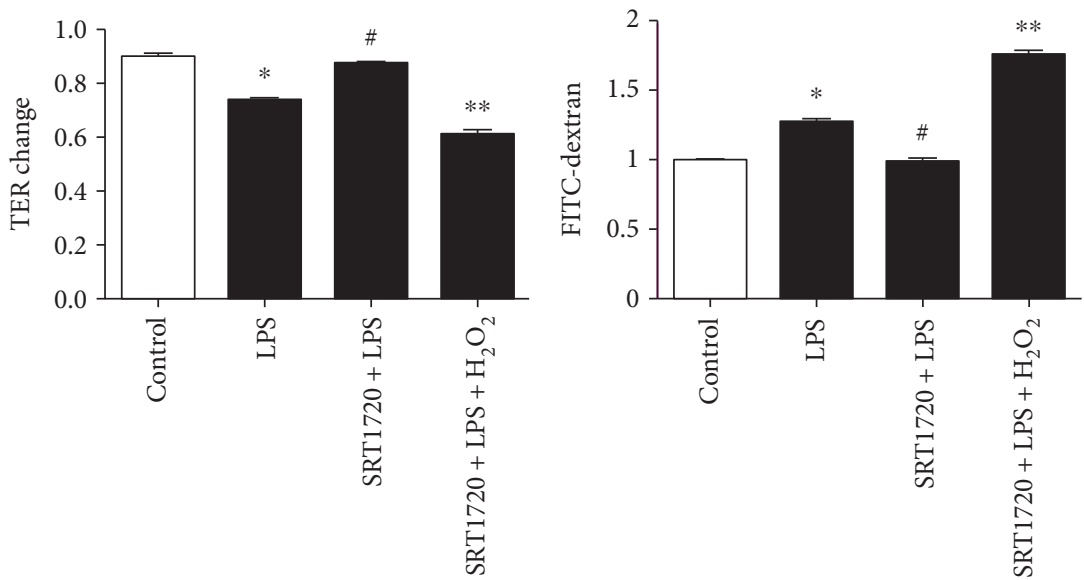

(c)

Figure 6: Effect of Sirt1 on the alteration of MDA, as well as on ROS generation in HUVECs. (a) SRT1720 abolished LPS-induced ROS generation. (b) SRT1720 attenuated LPS-induced MDA elevation. (c) $\mathrm{H}_{2} \mathrm{O}_{2}$ abolished the protective effect of SRT1720 on EC permeability. HUVECs were pretreated with or without SRT1720, then exposed to the control medium or LPS. Cells in the group SRT1720 + LPS $+\mathrm{H}_{2} \mathrm{O}_{2}$ were followed by $100 \mathrm{mM} \mathrm{H} \mathrm{O}_{2}$ treatment for $15 \mathrm{~min} . n=3{ }^{*} P<0.05$ versus control or control siRNA, ${ }^{*} P<0.05$ versus LPS or control siRNA + LPS, and ${ }^{* *} P<0.05$ versus SRT1720 + LPS.

(Figure 8(f)). All these data suggest that Sirt1-deacetylated p53 was probably engaged in the preservation of $\beta$-catenin expression to mitigate EC hyperpermeability induced by LPS.

\section{Discussion}

Sepsis represents a lethal health issue, not only relating to the complicated pathogenesis but also resulting in the prevalence of organ dysfunction and mortality. Though our realization of sepsis is ambiguous, we are still making progress in exploring it step by step. The development of sepsis is linked with hemodynamic alternations and microvascular barrier dysfunction to some degree. The disruption of vascular endothelial integrity results in capillary leakage and the loss of fluid. However, the mechanism underlying EC dysfunction caused by sepsis is still not clear. Our goal was to probe the relationship of LPS and the specific molecule Sirt1 in manipulating the function of the endothelial barrier. 

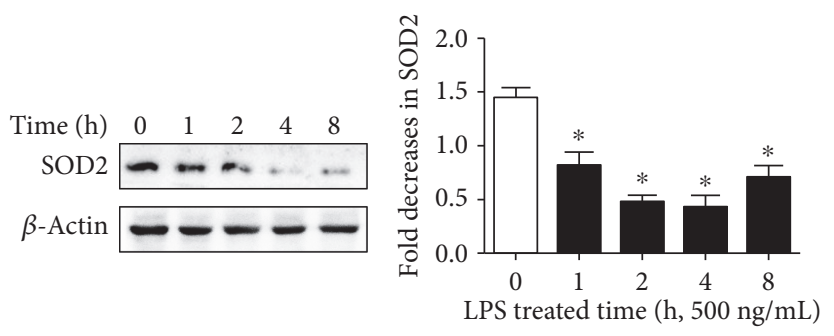

(a)
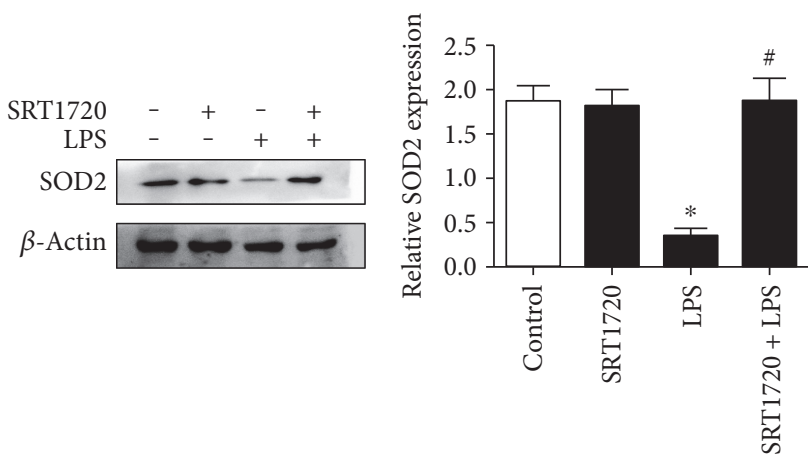

(c)
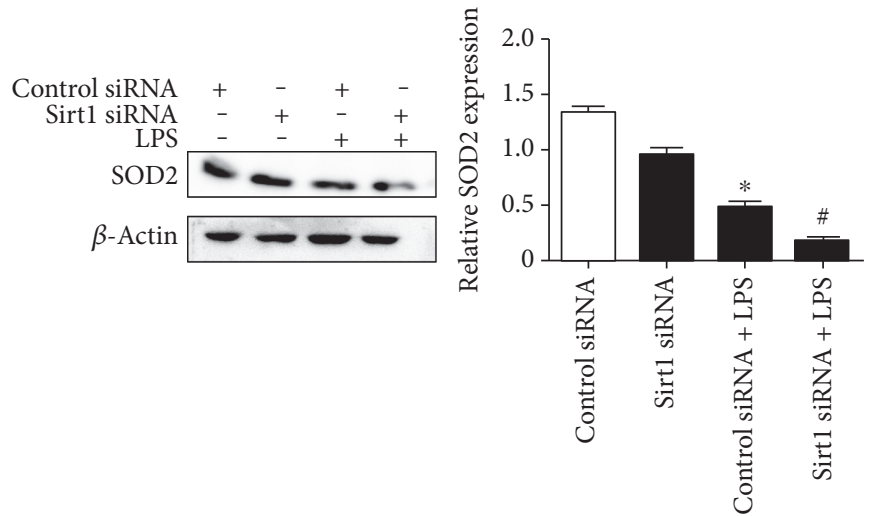

(e)
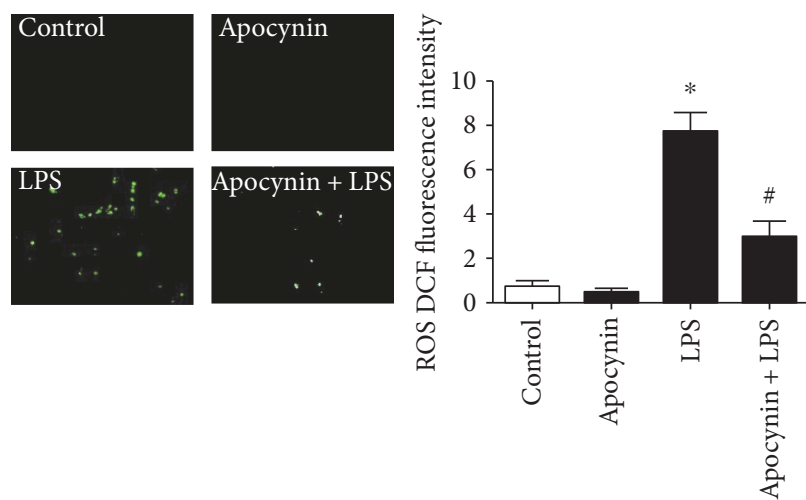

(g)

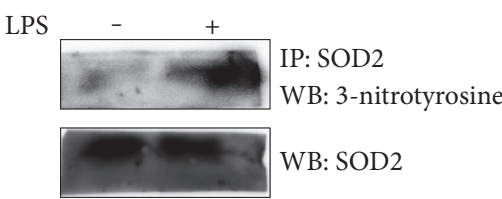

(b)

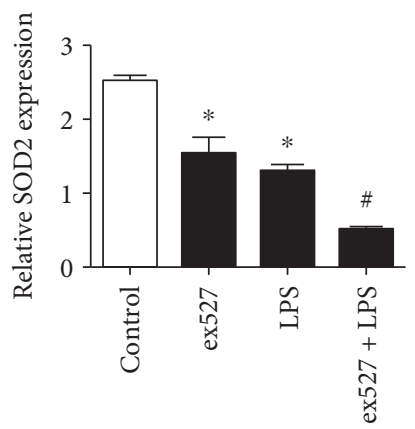

(d)
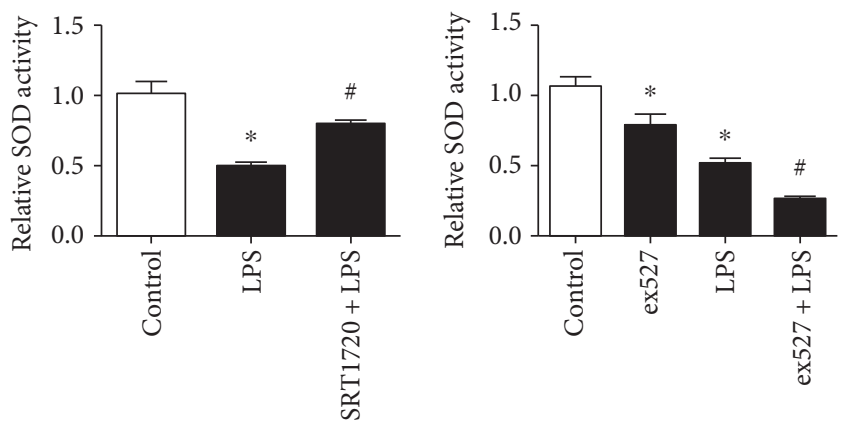

(f)

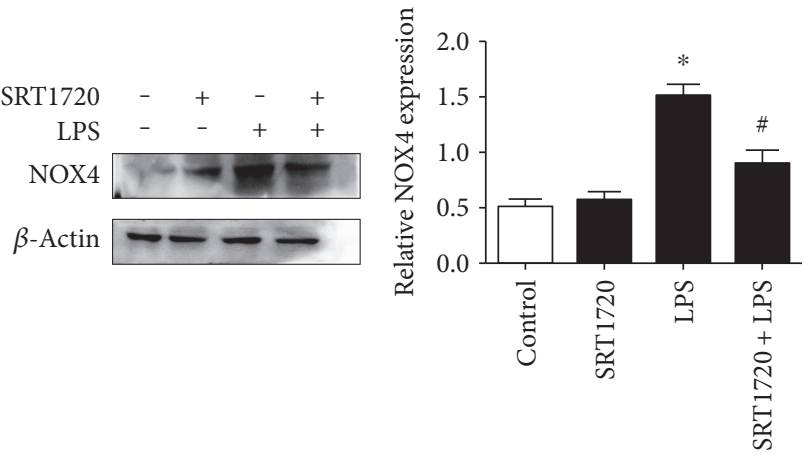

(h)

FIGURE 7: Effect of Sirt1 on the alteration of protein expression and activity of SOD2, as well as on NOX4 generation in HUVECs. (a) LPS evoked the decline in SOD2 expression in a time-dependent manner. (b) LPS induced tyrosine nitration of SOD2. (c-e) SRT1720 abolished the decrease in SOD2 expression upon LPS stimulation while ex527, as well as Sirt1 siRNA, duplicated the effect. (f) The Sirt1 activator reversed the LPS-induced decrease in SOD activity. ECs were treated with or without SRT1720 or ex 527 , followed by treatment of LPS $(500 \mathrm{ng} / \mathrm{mL}, 1 \mathrm{~h})$; then, SOD activity was detected using an SOD assay kit. (g) The NOX4 inhibitor apocynin (300 $\mu \mathrm{mol} / \mathrm{L}, 1 \mathrm{~h}$ ) attenuated LPS-induced ROS generation. ROS was measured through a fluorescent microscope. (h) SRT1720 abolished LPS-induced NOX4 elevation. $n=3 .{ }^{*} P<0.05$ versus control or control siRNA, ${ }^{*} P<0.05$ versus LPS or control siRNA + LPS. 


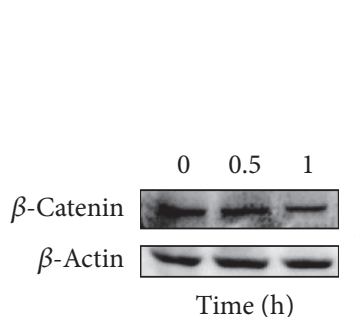

Time (h)

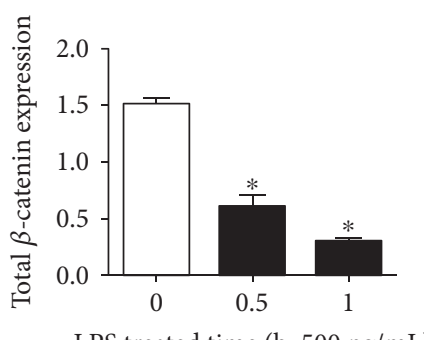

LPS treated time $(\mathrm{h}, 500 \mathrm{ng} / \mathrm{mL})$

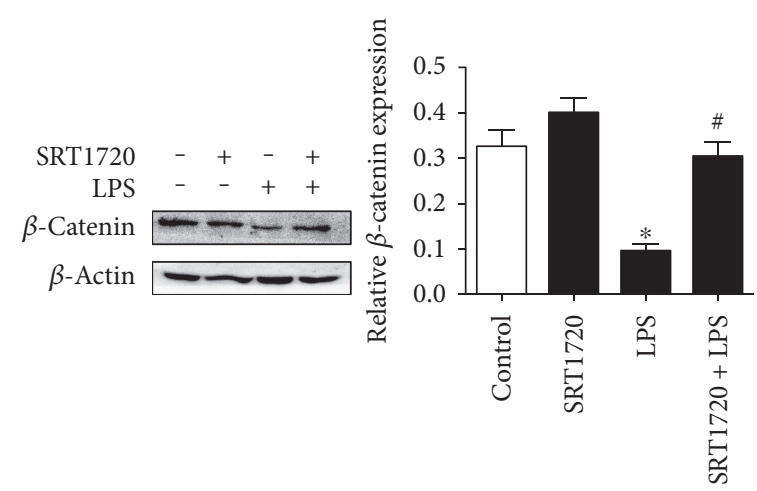

(b)

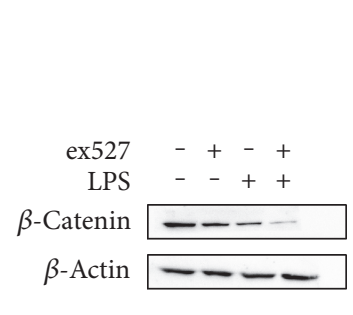

(a)

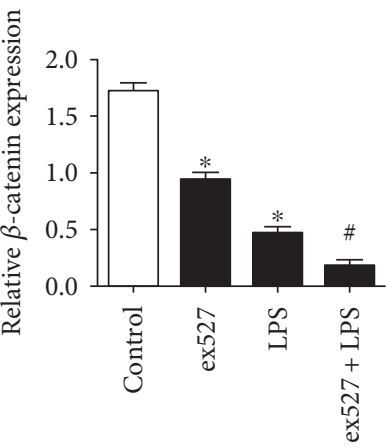

(c)
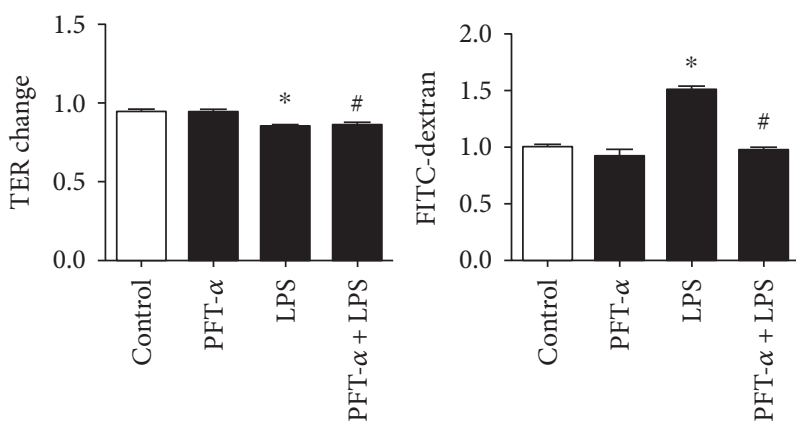

(e)

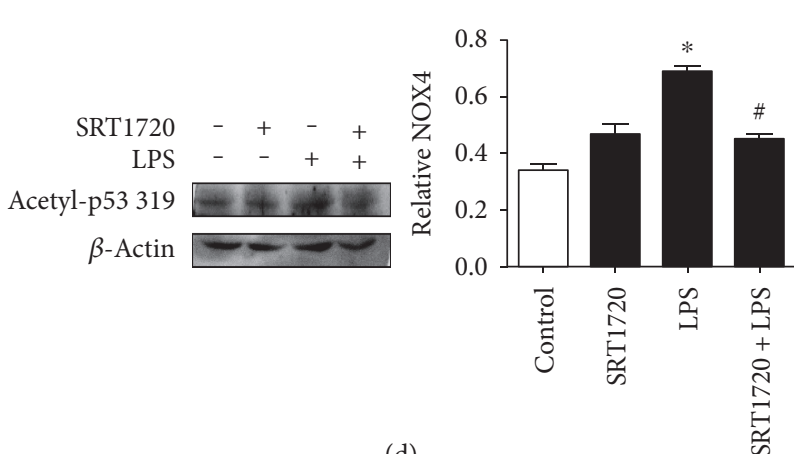

(d)

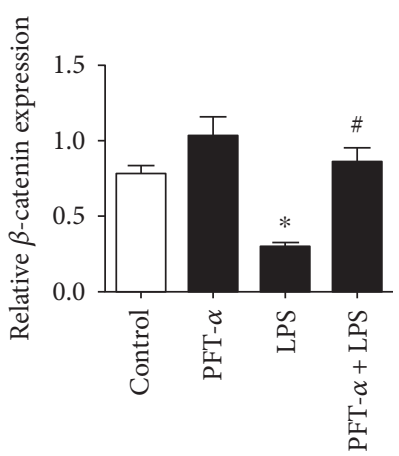

(f)

FIGURE 8: The role of SRT1720 and p53 in the downregulation of $\beta$-catenin induced by LPS in HUVECs. (a) Time-dependent changes in expression of $\beta$-catenin induced by $500 \mathrm{ng} / \mathrm{mL}$ LPS. (b) Pretreatment of SRT1720 prevented LPS-induced $\beta$-catenin downregulation. (c) Pretreatment of ex527 aggravated LPS-induced $\beta$-catenin downregulation. (d) SRT1720 attenuated LPS-evoked p53 319 acetylation. (e) p53 inhibition with PFT- $\alpha$ prevented LPS-induced EC hyperpermeability. (f) p53 inhibition with PFT- $\alpha$ prevented the LPS-induced decrease in $\beta$-catenin expression. $n=3{ }^{*} P<0.05$ versus control, ${ }^{\#} P<0.05$ versus LPS.

As an $\mathrm{NAD}^{+}$-dependent deacetylase, the longevity regulator Sirtl has gained great attention for its salutary effect on vascular function. Accumulating evidences have revealed that Sirt1 is connected closely with vascular disease and contributes to the modulation of key metabolic processes, including vascular ageing [29], ROS resistance [30], and apoptosis [31]. The present study revealed that Sirt1 protein was dramatically suppressed and sustained at a low level until $24 \mathrm{~h}$ in the application of LPS. It is ubiquitin that mediates a Sirt1 decrease. Substantial evidence shows that extreme stress response to sepsis induces remarkable drop of Sirt1 expression and hyperinflammation. Resolution of acute inflammation may rebalance Sirt1 and restore homeostasis [32]. Perhaps, ECs might firstly undergo a short time of Sirt1 inhibition in the early stage, and Sirt1 expression might be increased to defend inflammation and to keep homeostasis later. However, elevation of Sirt1 was not observed prior to $24 \mathrm{~h}$. Perhaps, prolonged time for stimulation should be conducted. In contrast, other studies showed that Sirt1 protein was elevated in human gingival fibroblasts [33]. There is no consensus on how Sirt1 was changed upon stress. The difference of Sirt1 response to LPS may be explained by different cell types and different conditions of LPS treatment. The mechanism and the regulation process are very complex, and further study remains to be explored. Given that Sirt1 exerts a protective effect on ECs, we speculated that Sirt1 might also ameliorate LPS-induced barrier dysfunction. In our study, we have demonstrated that LPS sparked EC 
hyperpermeability in a dose- and time-dependent manner. Inhibition of Sirt1 activity with a specific inhibitor or siRNA aggravated the barrier dysfunction, accompanied by F-actin rearrangement and VE-cadherin disruption, while elevation of its activity with an activator reversed the EC hyperpermeability, indicating the protective role of Sirt1 in LPS-induced EC dysfunction. Furthermore, treatment of LPS and Sirt1 activator at the same time could also reverse the hyperpermeability, indicating its remedial role in the EC barrier. Loss of the AJ protein $\beta$-catenin caused by LPS was also reversed due to upregulation of Sirt1 activity. Furthermore, in vivo experiment revealed that extravasation of FITC-labelled dextran from mesenteric venules was elevated in LPStreated mouse, while activation of Sirt1 with its agonist could counteract the exudation. These data illuminate an exciting avenue to further explore the potent molecular mechanism implicated in the beneficial role of Sirt1 in the protection of the EC barrier. And we speculate that Sirt1 might act as a critical node to respond to LPS-RAGE signaling pathways and to trigger the elevation of SOD2 and attenuation of NOX4 to resist ROS and the downregulation of p53 activity to prohibit the loss of the junctional protein, $\beta$-catenin.

LPS binding to RAGE is widely acknowledged [34, 35]. RAGE plays a pivotal role in mediating mortality after cecal ligation and puncture (CLP), the typical model of sepsis [36]. Interestingly, exogenous soluble RAGE (sRAGE), composed of the extracellular domain of RAGE, could block the response to LPS for its antagonist effect with the intracellular domain of RAGE. Furthermore, elevation of sRAGE was detected in septic patients compared with healthy volunteers, indicating that sRAGE could be a candidate as a new sepsis marker [37]. RAGE is critical in Sirt1 inhibition through the ubiquitin-proteasome pathway in diabetic nephropathy [38]. In the present study, we found that RAGE was engaged in LPS-induced downregulation of Sirt1 expression and activity in HUVECs. RAGE knockout mostly abrogated the LPS-sparked Sirt1 decrease and extravasation of mesenteric venules in a septic mouse model. Nevertheless, our result did not clarify the involvement of Sirt1 in the decrease in capillary leakage in $\mathrm{RAGE}^{-1-}$ mice. As we all know, in vivo study was characterized by the complexity of tissues and different cell types, including immune cells and other kinds of cells, which could not explain strongly the exact change of Sirt1 distributed in vessels, especially in the microvasculature. Thus, in our primary study shown in Figure 5(b), we isolated the PMVECs and were convinced that the decrease in Sirt1 expression induced by LPS was reversed in $\mathrm{RAGE}^{-/-}$cells, to some extent, indicating Sirt1 involvement in the decrease in capillary leakage in $\mathrm{RAGE}^{-/-}$mice. Ubiquitin-specific protease 22 (USP22) was reported to be associated with Sirt1 and can decrease Sirt1 ubiquitin. Furthermore, the AGEs-RAGEUSP22-Sirt1 has been elucidated as a cascade pathway that participated in the pathological progression of diabetic nephropathy. We also verified that LPS induced a Sirt1 decrease through ubiquitin pathways. Thus, we speculate that USP22 may also be the downstream of RAGE, which remains to be explored. Regarding other receptors for the ligand LPS, Toll-like receptor 4 (TLR4) was the first found LPS receptor and deficiency of TLR4 abated the LPS signaling pathway [39]. The present study found that a TLR4 inhibitor reversed an LPS-induced decrease in Sirt1 activity. These data revealed that RAGE and TLR4 were required for a decline in Sirt1 activity. To date, multiple studies have shown that RAGE and TLR4 could trigger similar inflammatory pathways in response to microbial products, including LPS [35, 40], high-mobility group protein 1 (HMGB1) [41, 42], and S100A8/A9 [43, 44]. Coactivation of RAGE and TLR4 was required to induce cellular responses when LPS was in complex with HMGB1 [45]. However, most evidences were focused on the downstream of RAGE and TLR4, and little was known about the crosstalk between them. It remains obscure whether RAGE and TLR4 combine with each other after LPS exposure. Both RAGE and TLR4 are comprised of three domains, including the extracellular domain, transmembrane domain, and intracellular domain. Perhaps, the structural and the biochemical factors of RAGE and TLR4 promote their cointeraction with LPS. Moreover, it is noteworthy that Toll-like receptor 2 (TLR2) serves as another crucial receptor of LPS, but it remains unknown whether TLR2 is engaged in response to LPS in vivo [46]. Hence, much more investigation should be warranted involving the potent role of TLR2 in an LPS-induced Sirt1 decline and EC hyperpermeability.

Growing evidence has proven that ROS render EC dysfunction, characterized by increasing fluid flux and inflammation reaction [47, 48]. Along with this, LPS-induced oxidative stress is closely related to lung vascular hyperpermeability and acute lung injury [49]. As a notable pioneer for scavenging ROS, SOD2 has been elucidated to contribute greatly to oxidative stress defense for decades [50]. As expected, we verified that the level and activity of SOD2 were remarkably decreased after exposure to LPS, consistent with the increase in ROS fluorescence by flow cytometry. However, the aforementioned effect was reversed by the upregulation of Sirt1, indicating Sirt1 as the activator of SOD2 to resist LPS-evoked ROS generation. In agreement with these findings, our previous research also revealed that the enhanced Sirt1 activity suppressed ROS by increasing expression of SOD2 to prevent hepatocyte mitochondrial dysfunction following hemorrhagic shock [51]. Furthermore, Sirt1 could increase transcription of SOD via deacetylating FOXO3a, hinting that the Sirt1/FOXO/SOD pathway may contribute to the suppression of ROS [17]. Another pathway, Sirt1peroxisome proliferator-activated receptor gamma coactivator (PGC-1 $\alpha$ ), is also potent to trigger SOD2 expression. Nevertheless, not only is the increase in SOD2 responsible for the reduced oxidative stress but the decrease in NOX4 is also critical in the suppression of ROS sources for the beneficial effect of Sirt1. Our study proved that it was the case. This was supported by the downregulation of NOX4 and the NOX4-driven ROS production after Sirt1 activation. The mechanism underlying Sirt1-dependent NOX4 inhibition may involve decreased PGC- $1 \alpha$ acetylation and the subsequent peroxisome proliferator-activated receptor- $\alpha$ (PPAR $\alpha)$ activation [20]. Hence, our finding illustrates the possible salutary role of Sirt1 in diminishing the LPS-induced ROS generation by elevating the expression and activity of SOD2 and attenuating NOX4 expression. Last but not the least, 
the mechanism by which Sirt1 upregulates SOD2 and downregulates NOX4 expression in an LPS-treated model still remains obscure. Thus, further exploration needs to be elucidated.

Intracellular junctional proteins have been elucidated to maintain the cell-cell barrier $[52,53]$. Degradation of the AJ protein $\beta$-catenin $[54,55]$ interferes with the sealing efficiency of ECs and results in barrier disruption. Our study revealed that activation of Sirt1 reversed LPS-sparked loss of $\beta$-catenin, while inactivation of Sirt1 exacerbated the effect. However, the mechanism was not clear. It has been demonstrated that downregulation of $\beta$-catenin could be evoked by activated p53 [24]. As p53 was the first found target of Sirt 1 and Sirt1 could inactivate p53 through deacetylating it in an LPS-treated model, we next determined whether p53 contributes to the LPS-induced loss of $\beta$ catenin. Our present study revealed that Sirt1 activation was required for p53 deacetylation and $\beta$-catenin upregulation. Furthermore, the p53 inhibitor ameliorated the LPSinduced downregulation of junction proteins and EC hyperpermeability, indicating the pivotal role of $\mathrm{p} 53$ in LPS-evoked EC dysfunction. Thus, it is likely that Sirt1 takes part in p53 inactivation, which is involved in the prevention of junction protein downregulation.

However, there are some limitations regarding experimental methods and designs. Firstly, we exposed mice to LPS injection intraperitoneally as the septic model. However, sepsis is not merely the event of gram-negative infection, and it refers to a complicated state characterized by a combination of gram-negative and gram-positive infection, involving systemic problems, including problems in the respiratory, urinary, gastrointestinal, and central nervous systems [56]. Hence, our model could not thoroughly explain the potent mechanism underlying sepsis-associated EC dysfunction, while the CLP model could. Nevertheless, at least, we are able to have a better understanding of the specific role played by gram-negative infection. Furthermore, the most crucial reason why we preferred LPS treatment to the CLP model is that CLP operation could probably ignite severe inflammation reaction and rise hyperpermeability of the mesenteric venules, which would arouse controversy surrounding the root cause of hyperpermeability in vivo, as to whether the injury was caused by CLP operation or by sepsis. So LPS treatment could avoid the ambiguity.

In summary, our present study revealed that Sirt1, as a hub, responds to LPS-RAGE signaling pathways and attenuates LPS-induced hyperpermeability through diminishing the generation of ROS and reversing the damage of junction proteins. Our finding sheds light on the possible protective role of Sirt1 in LPS-induced EC dysfunction and provides the possibility that Sirt1 could be a potent therapeutic target for the prevention and therapy of LPS-induced EC dysfunction.

\section{Methods}

4.1. Cells, Reagents, and Antibodies. HUVECs are purchased from ScienCell, and LPS was acquired from Sigma. SRT1720 (S1129), ex527 (S1514), and PFT- $\alpha$ (S2929) were purchased from Selleck (USA). Sirt1 siRNA (sc-40986) and control siRNA (sc-37007) were from Santa Cruz Biotechnology. 2,7-Dichlorofluoresein diacetate $\left(\mathrm{H}_{2} \mathrm{DCF}-\mathrm{DA}\right)$ was purchased from Sigma (USA). Antibodies against $\beta$-catenin (ab32572), against VE-cadherin (ab33168), and against SOD2 (ab13533) were from Abcam (USA). An antibody against Sirt1 (A0127) was from Abclonal (Boston, USA). An antibody against acetyl-p53 (K319) (YK0015) was from Immunoway (USA). An antibody against 3-nitrotyrosine (OM265454) was from OmnimAbs (USA). An antibody against p53 (WL01919) was from Wanleibio (China). An antibody against ubiquitin (3936S) was from CST (USA).

4.2. Assay for Quantification of Sirt1 Activity. Measurement of Sirt1 activity was performed using a Sirt1 activity assay kit (ab156065, Abcam). Cells were lysed and immunoprecipitated with a Sirt1 antibody $(10 \mu \mathrm{g})$. Afterwards, the reaction mixture containing $30 \mu \mathrm{L} \mathrm{ddH}_{2} \mathrm{O}, 5 \mu \mathrm{L}$ fluoro-substrate peptide, $5 \mu \mathrm{L} \mathrm{NAD}, 5 \mu \mathrm{L}$ developer, and $20 \mu \mathrm{L}$ sample was mixed thoroughly, and the fluorescence intensity was detected (ex. $350 \mathrm{~nm}$, em. $450 \mathrm{~nm}$ ) for 30 to 60 minutes at 1-2 min interval on a SpectraMax M5 system.

4.3. Western Blotting. Protein extracts from HUVECs or PMVECs were separated by SDS-PAGE and transferred to the PVDF membrane. The membrane was incubated with $5 \%$ BSA to block the nonspecific site for $1 \mathrm{~h}$ and incubated overnight at $4^{\circ} \mathrm{C}$ with primary antibodies for Sirt1, SOD2, $\beta$-catenin, acetyl-p53 (K319), p53, and 3nitrotyrosine at $1: 1000$. After using a secondary antibody for incubation for $1 \mathrm{~h}$, blots were visualized with the chemiluminescence method.

4.4. Measurement of Intracellular ROS Production. The level of ROS generation was assessed using peroxidesensitive dye $\mathrm{H}_{2}$ DCF-DA ( $\left.10 \mu \mathrm{M}, 30 \mathrm{~min}\right)$, followed by PBS washes for three times. Fluorescence of cells was detected through fluorescent microscopy or BD FACSVerse flow cytometry [57].

4.5. Detection of Superoxide Dismutase. According to the manufacturer's protocol, cells were collected with $0.25 \%$ trypsin and washed with PBS three times. Afterwards, the contents of SOD were detected using a Total Superoxide Dismutase Assay Kit with WST-8 (S0101, Beyotime), followed by concentration determination using ELISA kits.

4.6. Measurement of TER. As previously described [58], $200 \mu \mathrm{L}$ of cells mounting to $10^{5} / \mathrm{mL}$ were seeded onto the transwell upper chamber. The monolayer cellular barrier property was measured through detecting the TER value across the confluent ECs with EVOM ${ }^{2}$ (World Precision Instrument, USA). The value of the measured monolayer was expressed by the formula: TER $=$ TER of the experimental chamber minus TER of the cell-free chamber. Measurement was performed in triplicate and analyzed as percentage relative to TER level at time 0 .

4.7. Dextran Transendothelial Flux. As previously described [59], cells were grown to a confluent monolayer and exposed 
to different stimulations. The tracer FITC-labelled dextran $(1 \mu \mathrm{g} / \mathrm{mL})$ was added to the upper chamber to be incubated for 45 minutes. Later concentration of the dextran in the upper and bottom chamber was measured (ex. $488 \mathrm{~nm}$, em. $525 \mathrm{~nm}$ ) using an HST 7000 microplate reader.

4.8. Transfection of HUVECs with Sirt1 siRNA. HUVECs were cultured on 6-well plates until 30\%-50\% confluence and transfected with Sirtl siRNA and control siRNA using Lipofectamine 2000 transfection according to the manufacturer's protocol. After $48-72 \mathrm{~h}$, cells were exposed to different treatments.

4.9. Immunofluorescence Staining. HUVECs were cultured in confocal wells and subjected to different treatments. After cells were washed with PBS three times, they were fixed with $3.7 \%$ formaldehyde and perforated with $0.5 \%$ Triton X-100. Sequentially, ECs were blocked with 5\% BSA to prevent nonspecific conjunction and incubated with a primary antibody against VE-cadherin at $4^{\circ} \mathrm{C}$ overnight, followed by incubation with an FITC-conjugated rabbit antibody. For F-actin staining, ECs were treated with rhodamine phalloidin at room temperature for $1 \mathrm{~h}$. Cells were detected with a confocal scanning microscope (Zeiss, Germany) eventually.

4.10. PMVEC Isolation. As previously described [60], lungs of wild-type and $\mathrm{RAGE}^{-/-}$mice were isolated, sliced, and digested in collagenase type I for 45 minutes, followed by filtration through $70 \mu \mathrm{m}$ nylon filters and centrifugation at $300 \times \mathrm{g}$ for 10 minutes. Then, add appropriate CD31 to be incubated with the deposit and rotate thoroughly for 15 minutes. Afterwards, use the MACS separator to harvest the labelled cells.

4.11. LPS Treatment of Mice. Wild-type and RAGE ${ }^{-1-}$ C57 mice (18-20 g) were acquired from the lab center of Southern Medical University and Kanazawa University, respectively. The protocol using mice was approved by the Animal Care Committee of the Southern Medical University of China and was in accordance with the National Institutes of Health guidelines for ethical animal treatment. Mice were divided into four groups, including the saline group, LPS-treated group, SRT1720+LPS-treated group, and LPS-treated $\mathrm{RAGE}^{-/-}$group. Among these groups, mice in the LPStreated group were treated with $15 \mathrm{mg} / \mathrm{mL}$ LPS via intraperitoneal injection. Mice in SRT1720 + LPS group were treated with $10 \mathrm{mg} / \mathrm{kg}$ SRT1720 via tail vein injection $2 \mathrm{~h}$ prior to LPS injection. Mice were anesthetized via intramuscular injection with $13.3 \%$ ethyl carbamate plus $0.5 \%$ chloralose $(0.65 \mathrm{~mL} / \mathrm{kg})$ before carotid vein cannulation. The mice were placed on a platform to allow laparotomy incision and exposure of the appropriate mesenteric venules in the proximal ileum. Afterwards, $1 \mathrm{~mL}$ FITC-dextran $(100 \mathrm{mg} / \mathrm{mL})$ was administrated via the carotid vein firstly with $0.15 \mathrm{mg} / \mathrm{kg}$ / min dextran infusion constantly. Sequentially, fluorescence intensity was detected (ex. $488 \mathrm{~nm}$, em. $525 \mathrm{~nm}$ ), and the image was acquired in a fluorescence microscope (Nikon TE-300, Nikon Co., Japan). The formula calculating the permeability was as follows: $\triangle I=I_{\mathrm{o}} / I_{\mathrm{i}}$, where $I_{\mathrm{o}}$ indicates the intensity inside the vessel while $I_{\mathrm{i}}$ refers to the intensity outside the vessel $[61,62]$.

4.12. Statistical Analysis. The significance of variability was analyzed by one-way ANOVA. All data were presented as mean $\pm \mathrm{SE}$ from at least three dependent experiments. $P<0.05$ was considered to be significant.

\section{Conflicts of Interest}

The authors declare that there is no conflict of interests.

\section{Authors' Contributions}

Zhongqing Chen and Qiaobing Huang prepared the concept, designed the experiment, and revised the manuscript. Weijin Zhang, Yaoyuan Zhang, and Xiaohua Guo performed the research. Zhenhua Zeng, Jie Wu, Yanan Liu, Jing He, and Ruiting Wang compiled and analyzed the results. Weijin Zhang and Yaoyuan Zhang drafted the paper. Weijin Zhang and Yaoyuan Zhang contributed equally to this work. All authors approved the final manuscript.

\section{Acknowledgments}

This work was supported by the Natural Science Foundation of Guangdong Province (2016A030313561) for Zhongqing Chen, the National Natural Science Foundation of China (Grants 81370226, 81170297, and 0971201) for Qiaobing Huang, and the Guangdong Natural Science Foundation Team Project (S2013030013217).

\section{References}

[1] J. K. Chan, J. Roth, J. J. Oppenheim et al., "Alarmins: awaiting a clinical response," The Journal of Clinical Investigation, vol. 122, no. 8, pp. 2711-2719, 2012.

[2] M. M. Levy, M. P. Fink, J. C. Marshall et al., "2001 SCCM/ESICM/ACCP/ATS/SIS International Sepsis Definitions Conference," Critical Care Medicine, vol. 31, no. 4, pp. 1250-1256, 2003.

[3] G. Friedman, E. Silva, and J. L. Vincent, "Has the mortality of septic shock changed with time?," Critical Care Medicine, vol. 26, no. 12, pp. 2078-2086, 1998.

[4] S. Y. Yuan and R. R. Rigor, Regulation of Endothelial Barrier Function, Morgan and Claypool Life Sciences, San Rafael (CA), USA, 2010.

[5] N. M. Goldenberg, B. E. Steinberg, A. S. Slutsky, and W. L. Lee, "Broken barriers: a new take on sepsis pathogenesis," Science Translational Medicine, vol. 3, no. 88, pp. 25p-88p, 2011.

[6] D. De Backer, C. D. Orbegozo, K. Donadello, and J. L. Vincent, "Pathophysiology of microcirculatory dysfunction and the pathogenesis of septic shock," Virulence, vol. 5, no. 1, pp. 7379, 2014.

[7] H. Zhang and G. Y. Sun, "LPS induces permeability injury in lung microvascular endothelium via $\mathrm{AT}_{1}$ receptor," Archives of Biochemistry and Biophysics, vol. 441, no. 1, pp. 75-83, 2005.

[8] R. A. Frye, "Phylogenetic classification of prokaryotic and eukaryotic Sir2-like proteins," Biochemical and Biophysical Research Communications, vol. 273, no. 2, pp. 793-798, 2000. 
[9] P. Oberdoerffer, S. Michan, M. McVay et al., "SIRT1 redistribution on chromatin promotes genomic stability but alters gene expression during aging," Cell, vol. 135, no. 5, pp. 907918, 2008.

[10] J. Yu and J. Auwerx, "Protein deacetylation by SIRT1: an emerging key post-translational modification in metabolic regulation," Pharmacological Research, vol. 62, no. 1, pp. 3541, 2010.

[11] H. Ota, M. Akishita, M. Eto, K. Iijima, M. Kaneki, and Y. Ouchi, "Sirt1 modulates premature senescence-like phenotype in human endothelial cells," Journal of Molecular and Cellular Cardiology, vol. 43, no. 5, pp. 571-579, 2007.

[12] H. Z. Chen, Y. Z. Wan, and D. P. Liu, "Cross-talk between SIRT1 and p66Shc in vascular diseases," Trends in Cardiovascular Medicine, vol. 23, no. 7, pp. 237-241, 2013.

[13] A. Csiszar, N. Labinskyy, J. T. Pinto et al., "Resveratrol induces mitochondrial biogenesis in endothelial cells," American Journal of Physiology Heart and Circulatory Physiology, vol. 297, no. 1, pp. H13-H20, 2009.

[14] T. Li, J. Zhang, J. Feng et al., "Resveratrol reduces acute lung injury in a LPS-induced sepsis mouse model via activation of Sirt1," Molecular Medicine Reports, vol. 7, no. 6, pp. 18891895, 2013.

[15] X. Wang, N. L. Buechler, B. K. Yoza, C. E. McCall, and V. T. Vachharajani, "Resveratrol attenuates microvascular inflammation in sepsis via SIRT-1-induced modulation of adhesion molecules in ob/ob mice," Obesity, vol. 23, no. 6, pp. 12091217, 2015.

[16] C. Lisk, D. Kominsky, S. Ehrentraut et al., "Hemoglobininduced endothelial cell permeability is controlled part, in part, via a myeloid differentiation primary response gene-88dependent signaling mechanism," American Journal of Respiratory Cell and Molecular Biology, vol. 49, no. 4, pp. 619-626, 2013.

[17] T. Shimada, H. Furuta, A. Doi et al., "Des-acyl ghrelin protects microvascular endothelial cells from oxidative stress-induced apoptosis through sirtuin 1 signaling pathway," Metabolism, vol. 63, no. 4, pp. 469-474, 2014.

[18] N. E. de Picciotto, L. B. Gano, L. C. Johnson et al., "Nicotinamide mononucleotide supplementation reverses vascular dysfunction and oxidative stress with aging in mice," Aging Cell, vol. 15, no. 3, pp. 522-530, 2016.

[19] H. Chen, Y. Wan, S. Zhou et al., "Endothelium-specific SIRT1 overexpression inhibits hyperglycemia-induced upregulation of vascular cell senescence," Science China Life Sciences, vol. 55, no. 6, pp. 467-473, 2012.

[20] M. J. Zarzuelo, R. Lopez-Sepulveda, M. Sanchez et al., "SIRT1 inhibits NADPH oxidase activation and protects endothelial function in the rat aorta: implications for vascular aging," Biochemical Pharmacology, vol. 85, no. 9, pp. 1288-1296, 2013.

[21] B. Tharakan, J. Hellman, D. A. Sawant et al., “ $\beta$-Catenin dynamics in the regulation of microvascular endothelial cell hyperpermeability," Shock, vol. 37, no. 3, pp. 306-311, 2012.

[22] G. Bazzoni and E. Dejana, "Endothelial cell-to-cell junctions: molecular organization and role in vascular homeostasis," Physiological Reviews, vol. 84, no. 3, pp. 869-901, 2004.

[23] J. Yan, C. Chen, Q. Hu et al., "The role of p53 in brain edema after $24 \mathrm{~h}$ of experimental subarachnoid hemorrhage in a rat model," Experimental Neurology, vol. 214, no. 1, pp. 37-46, 2008.
[24] E. Sadot, B. Geiger, M. Oren, and A. Ben-Ze'Ev, "Down-regulation of $\beta$-catenin by activated p53," Molecular and Cellular Biology, vol. 21, no. 20, pp. 6768-6781, 2001.

[25] H. Vaziri, S. K. Dessain, E. E. Ng et al., " $h S I R 2^{\text {SIRT1 }}$ functions as an NAD-dependent p53 deacetylase," Cell, vol. 107, no. 2, pp. 149-159, 2001.

[26] E. Langley, M. Pearson, M. Faretta et al., "Human SIR2 deacetylates p53 and antagonizes PML/p53-induced cellular senescence," The EMBO Journal, vol. 21, no. 10, pp. 23832396, 2002.

[27] W. Yao, H. Zhao, R. Shi et al., "Recombinant protein transduction domain- $\mathrm{Cu} / \mathrm{Zn}$ superoxide dismutase alleviates bone cancer pain via peroxiredoxin 4 modulation and antioxidation," Biochemical and Biophysical Research Communications, vol. 486, no. 4, pp. 1143-1148, 2017.

[28] E. Lorenzo, C. Ruiz-Ruiz, A. J. Quesada et al., "Doxorubicin induces apoptosis and CD95 gene expression in human primary endothelial cells through a p53-dependent mechanism," The Journal of Biological Chemistry, vol. 277, no. 13, pp. 10883-10892, 2002.

[29] B. Bai, P. M. Vanhoutte, and Y. Wang, "Loss-of-SIRT1 function during vascular ageing: hyperphosphorylation mediated by cyclin-dependent kinase 5," Trends in Cardiovascular Medicine, vol. 24, no. 2, pp. 81-84, 2014.

[30] A. Salminen, K. Kaarniranta, and A. Kauppinen, "Crosstalk between oxidative stress and SIRT1: impact on the aging process," International Journal of Molecular Sciences, vol. 14, no. 2, pp. 3834-3859, 2013.

[31] Z. Zeng, Z. Chen, S. Xu et al., "Polydatin protecting kidneys against hemorrhagic shock-induced mitochondrial dysfunction via SIRT1 activation and p53 deacetylation," Oxidative Medicine and Cellular Longevity, vol. 2016, Article ID 1737185, 15 pages, 2016.

[32] V. T. Vachharajani, T. Liu, X. Wang, J. J. Hoth, B. K. Yoza, and C. E. McCall, "Sirtuins link inflammation and metabolism," Journal of Immunology Research, vol. 2016, Article ID 8167273, 10 pages, 2016.

[33] G. J. Park, Y. S. Kim, K. L. Kang et al., "Effects of sirtuin 1 activation on nicotine and lipopolysaccharide-induced cytotoxicity and inflammatory cytokine production in human gingival fibroblasts," Journal of Periodontal Research, vol. 48, no. 4, pp. 483-492, 2013.

[34] L. Wang, J. Wu, X. Guo, X. Huang, and Q. Huang, "RAGE plays a role in LPS-induced NF- $\kappa$ B activation and endothelial hyperpermeability," Sensors, vol. 17, no. 4, p. 722, 2017.

[35] Y. Yamamoto, A. Harashima, H. Saito et al., "Septic shock is associated with receptor for advanced glycation end products ligation of LPS," Journal of Immunology, vol. 186, no. 5, pp. 3248-3257, 2011.

[36] B. Liliensiek, M. A. Weigand, A. Bierhaus et al., "Receptor for advanced glycation end products (RAGE) regulates sepsis but not the adaptive immune response," The Journal of Clinical Investigation, vol. 113, no. 11, pp. 1641-1650, 2004.

[37] C. Bopp, S. Hofer, J. Weitz et al., "sRAGE is elevated in septic patients and associated with patients outcome," The Journal of Surgical Research, vol. 147, no. 1, pp. 79-83, 2008.

[38] K. P. Huang, C. Chen, J. Hao, J. Y. Huang, P. Q. Liu, and H. Q. Huang, "AGEs-RAGE system down-regulates Sirt1 through the ubiquitin-proteasome pathway to promote FN and TGF- $\beta 1$ expression in male rat glomerular mesangial cells," Endocrinology, vol. 156, no. 1, pp. 268-279, 2015. 
[39] K. Hoshino, O. Takeuchi, T. Kawai et al., "Cutting edge: Toll-like receptor 4 (TLR4)-deficient mice are hyporesponsive to lipopolysaccharide: evidence for TLR4 as the Lps gene product," Journal of Immunology, vol. 162, no. 7, pp. 3749-3752, 1999.

[40] A. Visintin, E. Latz, B. G. Monks, T. Espevik, and D. T. Golenbock, "Lysines 128 and 132 enable lipopolysaccharide binding to MD-2, leading to Toll-like receptor-4 aggregation and signal transduction," The Journal of Biological Chemistry, vol. 278, no. 48, pp. 48313-48320, 2003.

[41] O. Hori, J. Brett, T. Slattery et al., "The receptor for advanced glycation end products (RAGE) is a cellular binding site for amphoterin. Mediation of neurite outgrowth and coexpression of rage and amphoterin in the developing nervous system," The Journal of Biological Chemistry, vol. 270, no. 43, pp. 25752-25761, 1995.

[42] H. Yang, P. Lundback, L. Ottosson et al., "Redox modification of cysteine residues regulates the cytokine activity of high mobility group box-1 (HMGB1)," Molecular Medicine, vol. 18, pp. 250-259, 2012.

[43] O. Turovskaya, D. Foell, P. Sinha et al., "RAGE, carboxylated glycans and S100A8/A9 play essential roles in colitisassociated carcinogenesis," Carcinogenesis, vol. 29, no. 10, pp. 2035-2043, 2008.

[44] T. Vogl, K. Tenbrock, S. Ludwig et al., "Mrp8 and Mrp14 are endogenous activators of Toll-like receptor 4, promoting lethal, endotoxin-induced shock," Nature Medicine, vol. 13, no. 9, pp. 1042-1049, 2007.

[45] Y. H. Qin, S. M. Dai, G. S. Tang et al., "HMGB1 enhances the proinflammatory activity of lipopolysaccharide by promoting the phosphorylation of MAPK p38 through receptor for advanced glycation end products," Journal of Immunology, vol. 183, no. 10, pp. 6244-6250, 2009.

[46] M. Hirschfeld, Y. Ma, J. H. Weis, S. N. Vogel, and J. J. Weis, "Cutting edge: repurification of lipopolysaccharide eliminates signaling through both human and murine toll-like receptor 2," Journal of Immunology, vol. 165, no. 2, pp. 618-622, 2000.

[47] K. E. McQuaid and A. K. Keenan, "Endothelial barrier dysfunction and oxidative stress: roles for nitric oxide?," Experimental Physiology, vol. 82, no. 2, pp. 369-376, 1997.

[48] Y. H. Lei, P. J. Barnes, and D. F. Rogers, "Involvement of hydroxyl radicals in neurogenic airway plasma exudation and bronchoconstriction in guinea-pigs in vivo," British Journal of Pharmacology, vol. 117, no. 3, pp. 449-454, 1996.

[49] P. Fu, A. A. Birukova, J. Xing et al., "Amifostine reduces lung vascular permeability via suppression of inflammatory signalling," The European Respiratory Journal, vol. 33, no. 3, pp. 612-624, 2009.

[50] F. Johnson and C. Giulivi, "Superoxide dismutases and their impact upon human health," Molecular Aspects of Medicine, vol. 26, no. 4-5, pp. 340-352, 2005.

[51] P. Li, X. Wang, M. Zhao, R. Song, and K. S. Zhao, "Polydatin protects hepatocytes against mitochondrial injury in acute severe hemorrhagic shock via SIRT1-SOD2 pathway," Expert Opinion on Therapeutic Targets, vol. 19, no. 7, pp. 997-1010, 2015.

[52] Y. Komarova and A. B. Malik, "Regulation of endothelial permeability via paracellular and transcellular transport pathways," Annual Review of Physiology, vol. 72, pp. 463-493, 2010.
[53] D. Mehta and A. B. Malik, "Signaling mechanisms regulating endothelial permeability," Physiological Reviews, vol. 86, no. 1, pp. 279-367, 2006.

[54] O. O. Ogunshola, M. Moransard, and M. Gassmann, "Constitutive excessive erythrocytosis causes inflammation and increased vascular permeability in aged mouse brain," Brain Research, vol. 1531, pp. 48-57, 2013.

[55] D. Venkatesh, N. Fredette, B. Rostama et al., "RhoA-mediated signaling in Notch-induced senescence-like growth arrest and endothelial barrier dysfunction," Arteriosclerosis, Thrombosis, and Vascular Biology, vol. 31, no. 4, pp. 876-882, 2011.

[56] M. Singer, C. S. Deutschman, C. W. Seymour et al., "The third international consensus definitions for sepsis and septic shock (sepsis-3)," JAMA, vol. 315, no. 8, pp. 801-810, 2016.

[57] A. Aranda, L. Sequedo, L. Tolosa et al., "Dichloro-dihydrofluorescein diacetate (DCFH-DA) assay: a quantitative method for oxidative stress assessment of nanoparticletreated cells," Toxicology In Vitro, vol. 27, no. 2, pp. 954-963, 2013.

[58] S. Nonas, I. Miller, K. Kawkitinarong et al., "Oxidized phospholipids reduce vascular leak and inflammation in rat model of acute lung injury," American Journal of Respiratory and Critical Care Medicine, vol. 173, no. 10, pp. 1130-1138, 2006.

[59] J. H. Tinsley, M. H. Wu, W. Ma, A. C. Taulman, and S. Y. Yuan, "Activated neutrophils induce hyperpermeability and phosphorylation of adherens junction proteins in coronary venular endothelial cells," The Journal of Biological Chemistry, vol. 274, no. 35, pp. 24930-24934, 1999.

[60] W. Zhang, Q. Xu, J. Wu et al., "Role of Src in vascular hyperpermeability induced by advanced glycation end products," Scientific Reports, vol. 5, article 14090, 2015.

[61] T. M. Doggett and J. W. Breslin, "Acute alcohol intoxicationinduced microvascular leakage," Alcoholism, Clinical and Experimental Research, vol. 38, no. 9, pp. 2414-2426, 2014.

[62] J. W. Breslin, D. A. Daines, T. M. Doggett et al., "Rnd3 as a novel target to ameliorate microvascular leakage," Journal of the American Heart Association, vol. 5, no. 4, article e3336, 2016. 


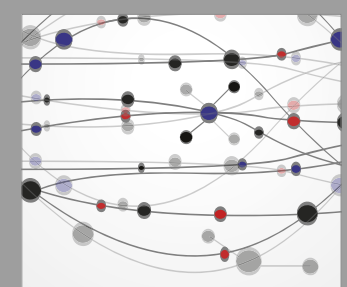

The Scientific World Journal
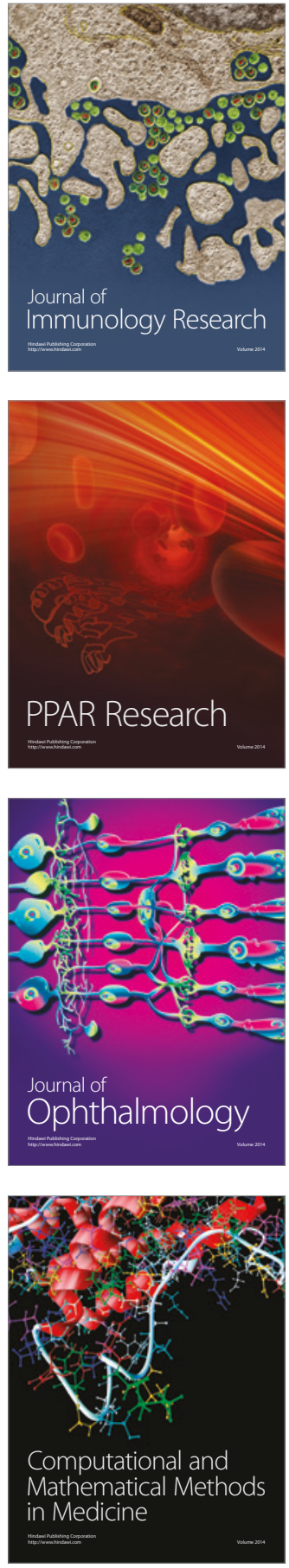

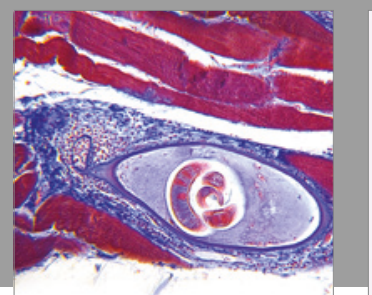

Gastroenterology Research and Practice
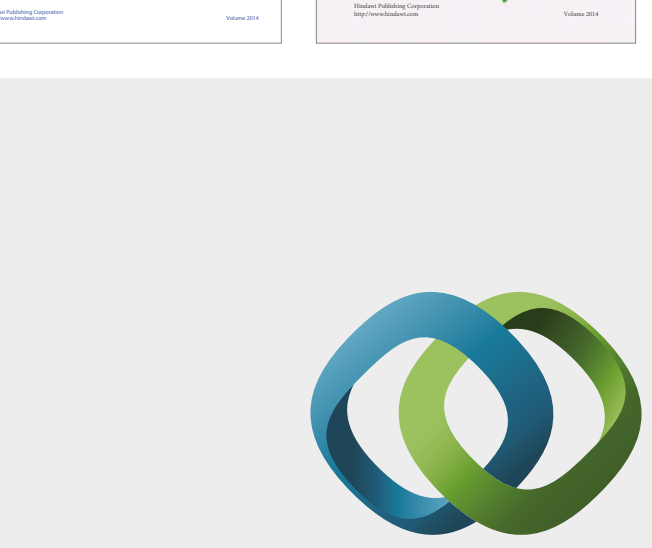

\section{Hindawi}

Submit your manuscripts at

https://www.hindawi.com
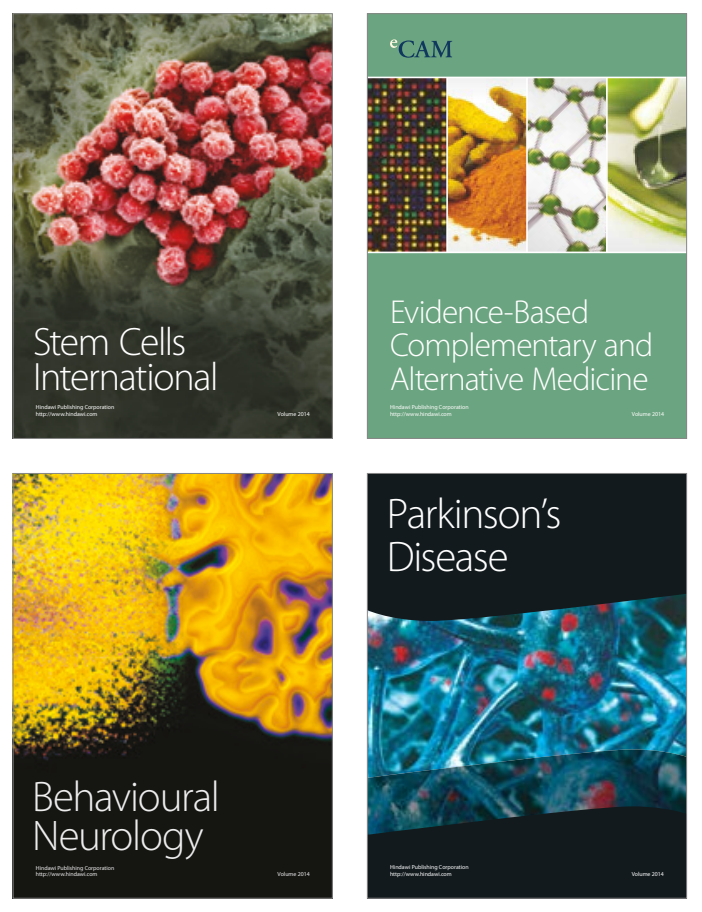
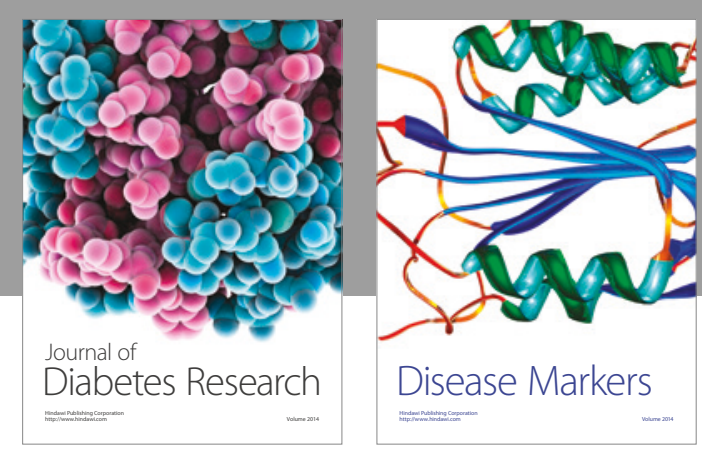

Disease Markers
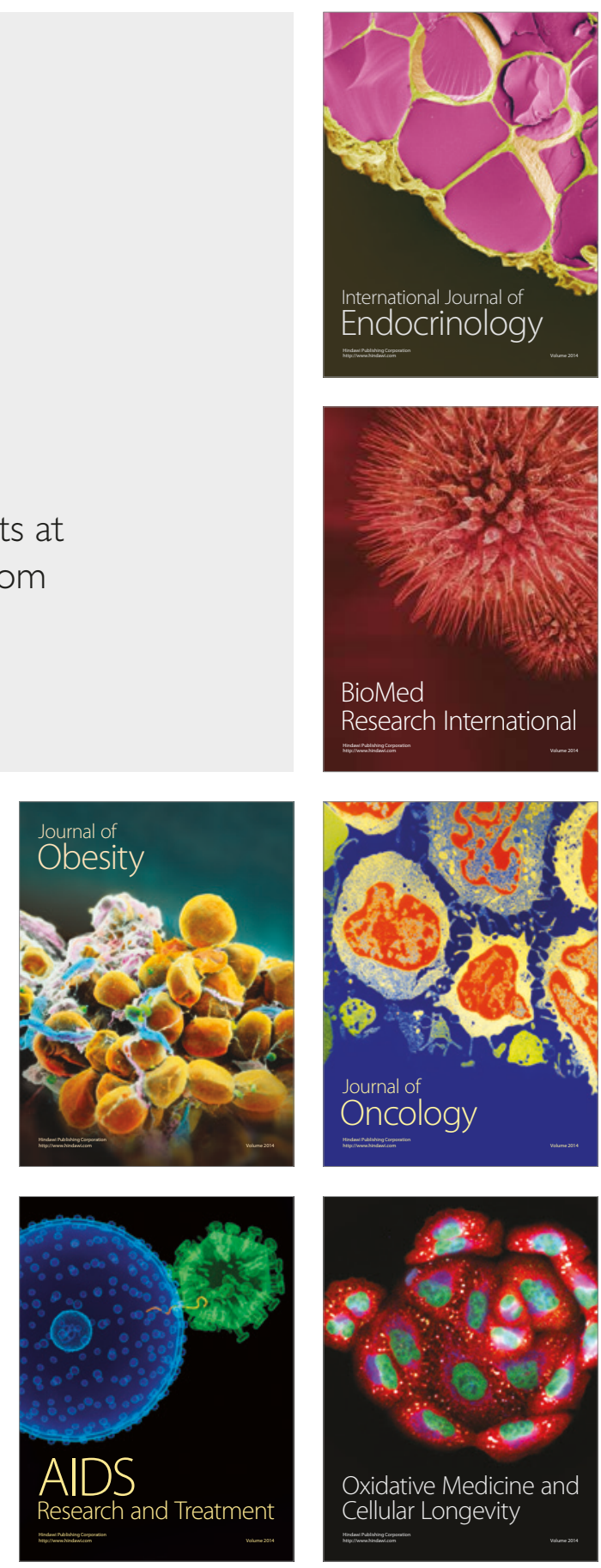